\title{
Stability of Begomoviral pathogenicity determinant $\beta C 1$ is modulated by mutually antagonistic SUMOylation and SIM interactions
}

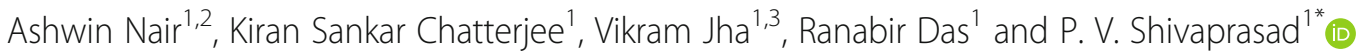

\begin{abstract}
Background: To successfully invade new hosts, plant viruses must break host resistance and be competent to move within and between plant cells. As a means, viral proteins known as pathogenicity determinants have evolved to coordinate a network of protein interactions. The $\beta C 1$ protein encoded by specific geminiviral satellites acts as a key pathogenicity determinant for this disease-causing family of plant viruses. Post-translational modifications (PTMs) such as ubiquitination and phosphorylation of the $\beta C 1$ protein have been shown to occur in diverse viruses. However, the relevance of these and other layers of PTMs in host-geminiviral interactions has not been fully understood.

Results: Here we identified the significance of a novel layer of PTMs in the $\beta C 1$ protein of Synedrella yellow vein clearing virus (SyYVCV), a newly identified member of the Begomovirus genus of Geminiviruses. This protein has conserved SUMOylation and SUMO-interacting motifs (SIMs), and we observed SUMOylation of SyYVCV $\beta C 1$ in host plants as a defensive strategy against ubiquitin-mediated degradation. Counteracting this, SIMs encoded in $\beta C 1$ mediate the degradation of $\beta C 1$; however, both these PTMs are essential for the function of $\beta C 1$ protein since SIM and SUMOylation motif mutants failed to promote pathogenicity and viral replication in vivo. SUMOylation in different motifs of $\beta C 1$ led to functionally distinct outcomes, regulating the stability and function of the $\beta C 1$ protein, as well as increased global SUMOylation of host proteins.
\end{abstract}

Conclusion: Our results indicate the presence of a novel mechanism mediating a fine balance between defence and counter-defence in which a SIM site is competitively sought for degradation and, as a counter-defence, $\beta C 1$ undergoes SUMOylation to escape from its degradation.

Keywords: $\beta C 1$ protein, SUMOylation, SIM interaction, Begomovirus, Host-pathogen interactions, Post-translational modification, NMR

\footnotetext{
* Correspondence: shivaprasad@ncbs.res.in

${ }^{1}$ National Centre for Biological Sciences, Tata Institute of Fundamental

Research, GKVK Campus, Bellary Road, Bangalore 560065, India

Full list of author information is available at the end of the article
}

C C The Author(s). 2020 Open Access This article is licensed under a Creative Commons Attribution 4.0 International License, which permits use, sharing, adaptation, distribution and reproduction in any medium or format, as long as you give appropriate credit to the original author(s) and the source, provide a link to the Creative Commons licence, and indicate if changes were made. The images or other third party material in this article are included in the article's Creative Commons licence, unless indicated otherwise in a credit line to the material. If material is not included in the article's Creative Commons licence and your intended use is not permitted by statutory regulation or exceeds the permitted use, you will need to obtain permission directly from the copyright holder. To view a copy of this licence, visit http://creativecommons.org/licenses/by/4.0/. The Creative Commons Public Domain Dedication waiver (http://creativecommons.org/publicdomain/zero/1.0/) applies to the data made available in this article, unless otherwise stated in a credit line to the data. 


\section{Background}

Viruses are obligate intracellular pathogens overcoming host defence as a means of survival. During millions of years of evolution, many strategies have been evolved by both the host and the virus to counter each other [1]. The dependence of viruses on host resources, along with the variations in the host defence, results in acute susceptibility, chronic infections or resistance. Within the constraints of a small genetic material, viruses code for essential proteins that are mostly multifunctional, playing critical roles in viral replication, packaging and counterdefence. A few proteins termed as pathogenicity determinants have a special place since they are essential to counter host defences, thereby playing crucial roles in viral infection. Often, viruses may replicate without these proteins, but are unable to mount a systemic infection, eventually being subdued by the host defence system [2-6].

The host has evolved and modified its innate cellular pathways to detect and neutralize various pathogenic threats apart from maintaining regular cellular homeostasis [7]. Post-translational modification (PTM) of proteins diversifies their functions as well as offering an additional regulation over their cellular activity. The host has evolved its PTM machinery to modify and subdue incoming pathogenic proteins to affect pathogenicity [7]. For example, the host recognizes and directs phosphorylation of Turnip yellow mosaic virus (TYMV) RNAdependent DNA polymerase (RdRp) protein tagging it for degradation, thus terminating viral genome replication [8]. Similarly, phosphorylation of Tomato yellow leaf curl virus (TYLCV) $\beta C 1$ by host SnRK1 kinase results in its inactivation [9]. Another common and effective strategy involves ubiquitination of viral proteins for proteasome-mediated degradation. Due to their roles in the intracellular and intercellular movement, often exposing themselves to host factors, it is likely that movement proteins (MP) are optimal targets for hostmediated degradation [10]. For example, MP from Tobacco mosaic virus (TMV), Cotton leaf curl Multan virus (CLCuMuV $\beta C 1)$ and Tomato yellow leaf curl China virus (TYLCCNV $\beta C 1$ ) are direct targets for host ubiquitination leading to their degradation [11-14]. It is proposed that viruses are masters in remodelling cellular systems for their exploitation, including the highjacking of the host PTM machinery for their counter-defence and infection. In agreement with this, many geminiviral proteins require PTMs for their activity as seen in Tomato leaf curl Yunnan virus (TLCYnV C4) and Cabbage leaf curl virus (CaLCuV NSP) proteins that require phosphorylation for their activity $[15,16]$.

Many studies link PTMs such as ubiquitination, phosphorylation and SUMOylation as major elements of the
PTM-derived regulation of cellular homeostasis and defence [17]. Ubiquitination (Ub) is a versatile PTM with up to eight different kinds of poly-Ub linkages leading to distinctive outcomes of the substrates. For example, K48 poly-ubiquitination is a major signal for proteasomal degradation [18], whereas K63 poly-ubiquitin linkage mediates cellular processes such as localization, DNA repair and autophagy [19]. Phosphorylation of proteins on the other hand is an addition of a charged and hydrophilic phosphoryl group into the side chain of amino acids, possibly changing the structure and function of the target protein [20]. SUMOylation too plays diverse roles such as in DNA repair sensing, stress response, indirect tagging of proteins for degradation and altering the subcellular localization of various proteins [21]. It is a highly dynamic transient modification involving a covalent addition of small ubiquitin-like moiety onto lysine residues of the substrate via an isopeptide linkage. The transient nature of SUMOylation is due to the presence of enzymes called Sentrin-specific protein proteases (SENPs) that are necessary for the maturation of SUMO, as well as the removal of linked SUMO from substrates via cleavage of isopeptide bond [22].

The ATP-dependent conjugation of SUMO involves recognition of the C-terminal di-glycine motif (GG) of SUMO protein by SUMOylation enzyme E1 which is a heterodimer of SAE1/SAE2 in Arabidopsis (AOS1/UBA2 mammals). A thioester bond formation leads to the linkage of SUMO with E1 which is then transferred to the conjugating enzyme SCE1 (homolog of UBC9 in mammals). SCE1 in an E3-dependent or E3-independent manner transfers the SUMO moiety by a covalent isopeptide linkage to the lysine residue of the target protein having a consensus sequence $\psi-\mathrm{K}-\mathrm{X}-\mathrm{E} / \mathrm{D}$ (where ' $\psi$ ' represents a hydrophobic amino acid and ' $\mathrm{X}$ ' represents any amino acid) $[23,24]$. In conjunction with SUMOylation, another non-covalent interaction occurs on the SUMOinteracting motifs (SIMs) of the same protein. Almost all the proteins known to undergo SUMOylation have SIM sites, highlighting the importance of SIM in the SUMOylation process [25]. Interestingly, all examples of non-consensus SUMOylation occurring on lysines without a consensus SUMO motif indicate the importance of a functional SIM motif for SUMOylation [26]. Mechanistically, SIM sites function either by docking the conjugation enzyme close to the SUMOylation site or by locally increasing the concentration of SUMO moieties close to the consensus lysine leading to efficient SUMOylation [27]. In addition to the effect on SUMOylation, SIM sites can act as docking sites for other SUMOylated proteins, increasing the repertoire of cellular interactions [25]. Unlike ubiquitination, whose conjugation process depends on multiple E2 enzymes, SUMOylation uses a single E2 in combination with 
multiple E3s. SUMOylation and SIM interaction both lead to variable outcomes, affecting protein localization, stability and interactions with other partner proteins.

SUMOylation being a versatile PTM is used by plants as regulators of major pathways. For example, SUMOylation of DNA repair proteins upon DNA damage acts as the trigger for the assembly of proteins on the damage site [28]. In plants, SUMOylation of cellular defence switches acts as triggers for antiviral defence [17]. SUMOylation mediated by E3 SUMO ligase SIZ1 has been implicated in a negative regulation of salicylic acid (SA)-based defence signalling thereby regulating expression of pathogenesis-related (PR) genes [29]. In addition, SUMOylation-mediated disruption of defence regulators is a crucial step during viral infection. Several RNA viral proteins undergo SUMOylation upon infection. For example, $\mathrm{NiB}$ protein of Turnip mosaic virus undergoes SUMOylation leading to relocalization of SUMO nuclear pool, causing inactivation of defence regulators such as NPR1 [30].

Geminiviral satellite DNA-coded $\beta C 1$ is a small protein of approximately $13-15 \mathrm{kDa}$. It is an important viral protein that counters various host defence mechanisms as well as acting as a movement protein in monopartite begomoviruses. It is also a viral suppressor of RNA silencing (VSR) of host silencing defence machinery and is shown to bind different nucleic acids [31]. It has been well documented that TYLCCNV $\beta C 1$ gets degraded in host cells [13]. However, it is not clear if this degradation is a host defence mechanism or a viral strategy to regulate the expression of this protein in the infected cells. The latter idea is supported by the evidence that a relatively higher expression of $\beta C 1$ protein is toxic to cells [32]. In line with this, overexpression of $\beta C 1$ and other movement-associated proteins cause developmental defects when expressed as transgenes. Further, CLCuMuB $\beta C 1$ interacts with host autophagy machinery. Perturbing interaction of CLCuMuB $\beta C 1$ with autophagy regulator led to premature death of host thereby reducing viral propagation. These studies suggest a possible co-evolution between compatible host and the virus, leading to late or dormant infection [14].

In this study, we elucidated novel PTMs on Synedrella yellow vein clearing virus (SyYVCV) pathogenicity determinant $\beta C 1$ protein. We identified the roles of SUMOylation motifs and SIMs of $\beta C 1$ during viral infection. Using in vitro and in vivo methods, we show that $\beta C 1$ undergoes SUMOylation in plants. Furthermore, using NMR and in vivo studies, we show that $\beta C 1$ SIMs are responsible for its degradation, while SUMOylation of different sites in $\beta C 1$ leads to different outcomes deciding the fate of $\beta C 1$ proteins. We also show a potential way by which geminiviral $\beta C 1$ can interact and subdue host defence response mediated by SUMOylation. Our results indicate the presence of a novel mechanism operating during plant-virus interactions involving PTMs, leading to the fine balance between function and stability on the one hand or inactivity and degradation on the other.

\section{Results \\ SyYVCV $\beta C 1$, a geminiviral pathogenicity determinant, undergoes SUMOylation in host plants}

SyYVCV is a new monopartite Begomovirus recently characterized by our group, having a $2.7-\mathrm{kb}$ DNA A component and a satellite DNA $\beta$ of $1.3 \mathrm{~kb}$ length [33]. It causes vein clearing disease in its natural host and leaf curling in Nicotiana tabacum. SyYVCV $\beta C 1$ is 118 aa long protein with multiple intrinsically disordered regions coded by the only ORF known in DNA $\beta$. Using bioinformatics tools (GPS SUMO, JASSA) [26, 34], we identified three putative SUMOylation sites spread throughout the length of the protein (Fig. 1a). Among the three predicted SUMOylation sites (Ss), Ss1 (Lysine, K18) and Ss2 (Lysine, K24) showed inverted SUMOylation consensus $(\mathrm{D} / \mathrm{EXK} \psi)$, whereas Ss3 (K83) was predicted to have a consensus site for SUMOylation ( $\psi \mathrm{KXX} / \mathrm{D})$ with a low score (Additional file 1: Fig. S1A). Both inverted and consensus sites usually get SUMOylated [35].

Among the three predicted sites, Ss1 (K18) was the most conserved SUMOylation site (77\% of all $\beta \mathrm{C} 1$ entries from the nr Uniprot database) when compared between viruses that are associated with $\beta$ DNA. This was followed by Ss3 (K83; 37\%). Ss2 (K24) was least conserved among all predicted sites (10\%), mostly restricted to SyYVCV and AYVV (Ageratum Yellow Vein Virus) cluster of viruses that result in vein clearing symptoms (Fig. 1a, Additional file 1: Fig. S1B and Additional file 2: Table S1).

To validate whether SyYVCV $\beta C 1$ is a direct target for SUMO conjugation, an in vitro SUMOylation assay containing recombinant $E$. coli-purified MBP- $\beta C 1$, SUMOactivating enzyme mixture (E1 homolog), Ubc9 (E2 homolog) and His-tagged SUMO1 (NbSUMO1-GG, Cterminal activated SUMO1 $N$. benthamiana) was performed. We observed multiple slow-migrating high molecular weight intermediate products in a denaturing PAGE gel when blotted with anti-AtSUMO1 antibody only in the presence of $\beta C 1$ as substrate. These bands were detected only in the presence of $\mathrm{Mg}^{2+}$-ATP, and likely represented a SUMOylated $\beta C 1$ (Fig. 1b). The SUMOylated products were a result of the direct reaction involving E1 and E2, since the absence of E1 and E2 in the reaction did not produce higher-order bands (Additional file 1: Fig. S2A). These results likely suggested the SUMOylation of $\beta C 1$ in vitro in an E1, E2catalysed ATP-dependent reaction. To further validate 


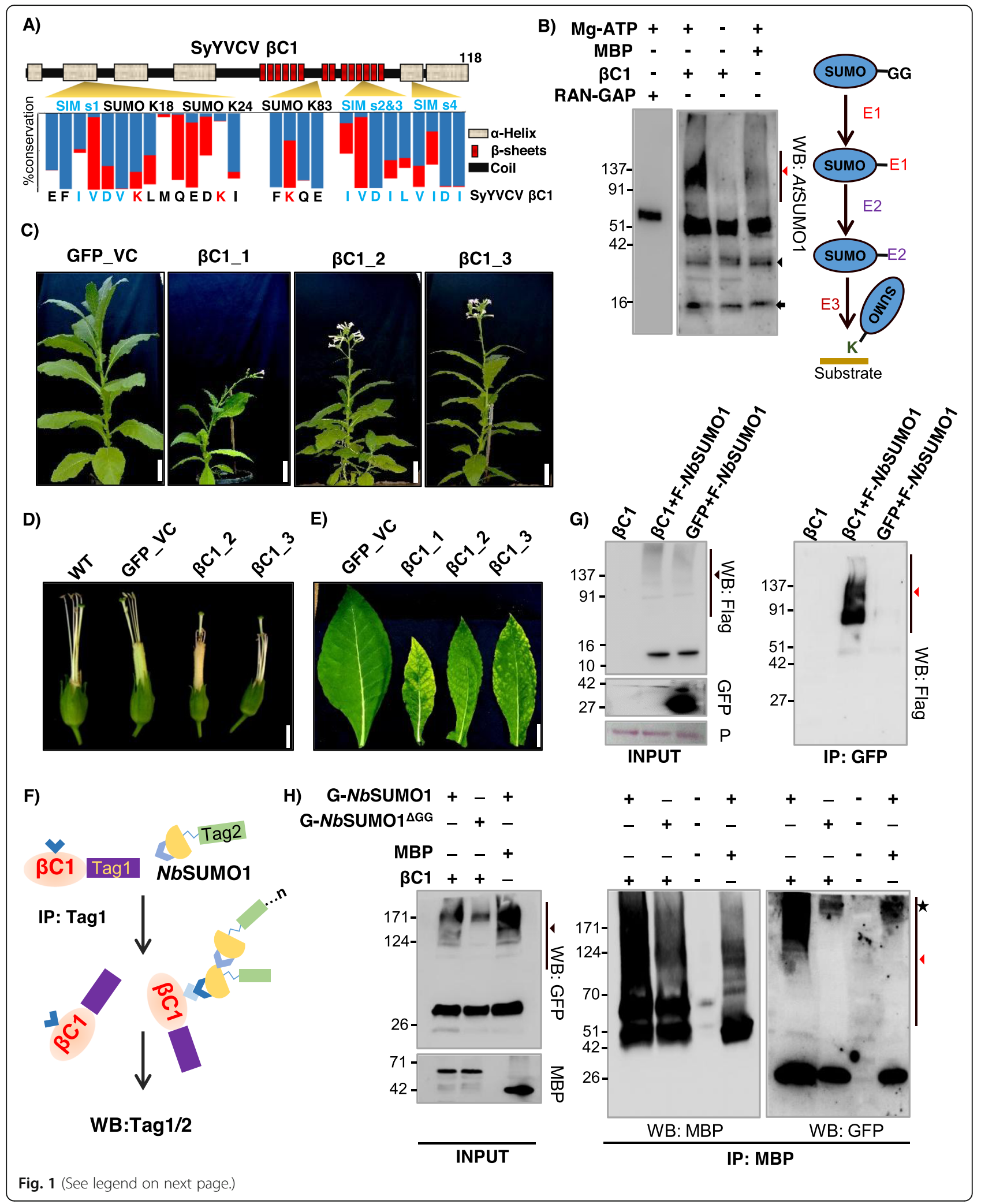


(See figure on previous page.)

Fig. $1 \beta C 1$ undergoes SUMOylation in vitro and in vivo. a Schematic showing predicted SUMOylation and SIM sites. Secondary structure was predicted using Predictprotein software. Blue bar indicates the percentage of amino acid conservation between $\beta C 1$ sequences. The red bar shows the presence of structurally similar residue substitutions. b Left panel: in vitro SUMOylation with MBP- $\beta C 1(59 \mathrm{kDa}), \mathrm{MBP}(42 \mathrm{kDa})$ and RanGap (63 kDa, positive control) using purified SUMO conjugating enzymes. Right panel: schematic of SUMOylation cascade. Red and black triangles represent poly-SUMOylated substrates and E1-SUMO conjugate, respectively. The black arrow indicates NbSUMO1. c Phenotype of transgenic N. tabacum lines overexpressing eGFP-tagged $\beta C 1$. d Flower phenotype (without petals). e Symptomatic leaves of transgenic $\beta C 1$ plants. f Diagram showing the Co-IP presented in $\mathbf{g}$ and $\mathbf{h}$. $\mathbf{g}$ Co-IP of transiently expressed GFP- $\beta C 1$ ( $42 \mathrm{kDa}$ ) and vector GFP with co-expressed Flag-tagged NbSUMO1 (F-NbSUMO1). h Co-IP of transiently expressed MBP-BC1 (59 kDa) with co-expressed GFP-tagged NbSUMO1 and $\mathrm{NbSUMO}^{\triangle \mathrm{GG}}$. Size bars in $\mathbf{c}, \mathbf{d}$ and $\mathbf{e}$ are $10,0.8$ and $3 \mathrm{~cm}$, respectively. Black and red triangles indicate NbSUMO1 poly-SUMOylated proteins and NbSUMO1- $\beta C 1$ conjugates, respectively. The asterisk indicates a non-specific band. P, Ponceau staining showing RUBISCO large subunit. Protein sizes are shown in $\mathrm{kDa}$

this interaction, we performed yeast two-hybrid $(\mathrm{Y} 2 \mathrm{H})$ assay with $N b S U M O 1$ as prey and $\beta C 1$ as bait. We observed the growth of yeast cells, suggesting an interaction between $\mathrm{NbSUMO} 1$ and $\beta \mathrm{C} 1$ (Additional file 1 : Fig. S2B).

To understand the biological function of $\beta \mathrm{C} 1 \mathrm{SUMOy}-$ lation, we generated transgenic $N$. tabacum plants overexpressing $\beta C 1$. As expected and observed in case of overexpression of many pathogenicity determinants, overexpression of $\beta C 1$ induced symptoms similar to virus-infected plants [36] (Fig. 1c, d, e, and Additional file 1: Fig. S3). These plants exhibited abnormal phenotypes such as stunted growth, early flowering, pointed leaves, shorter internodes, branching and mosaic patches on the leaves. In addition to these phenotypes, the expression of $\beta C 1$ induced exerted stigma phenotype and seed sterility. Although the expression of a DNA viral protein in plants was not previously reported to show exerted stigma phenotype, RNA viral proteins are known to produce such defects [37].

To substantiate the defects observed in transgenic plants overexpressing $\beta C 1$ was because of the interactions mediated by $\beta C 1$ SIM and SUMOylation motifs, we verified whether $\beta C 1$ undergoes SUMOylation in planta, we performed co-immunoprecipitation (Co-IP) of $\beta \mathrm{C} 1$ from stable transgenic plants overexpressing $\beta C 1$. $\beta \mathrm{C} 1$, but not control GFP expressing plants, showed high molecular weight bands when blotted with anti-GFP (Additional file 1: Fig. S4A) and anti-AtSUMO1 antibodies (Additional file 1: Fig. S4B). Since SUMOylation is a dynamic process and less than $1 \%$ of any substrate protein is SUMOylated at a given point in cells [21], we transiently overexpressed $3 \mathrm{X}$ Flag-tagged NbSUMO1 and GFP-tagged $\beta C 1$ in $N$. tabacum to increase the chance of $\beta C 1$ SUMOylation and its detection through WB (Fig. 1f). Co-IP of $\beta C 1$ was performed, followed by western blot analysis with anti-FLAG antibody. Any signal from the pull-down products after blotting with anti-FLAG antibody essentially indicates an interaction of $\mathrm{NbSUMO} 1$ with $\beta \mathrm{C} 1$ (Fig. 1g). After Co-IP with anti-GFP and detection with anti-FLAG antibody, we observed signals ranging from 60 to $150 \mathrm{kDa}$ only in $\beta C 1$ pull-down products, but not in control, indicating the presence of SUMO-conjugated $\beta C 1$ (Fig. 1g). SUMOylation of $\beta C 1$ produced higher-order intermediates likely due to SUMOylation of multiple SUMO conjugation sites of $\beta \mathrm{C} 1$ as well as poly-SUMOylation of NbSUMO1 conjugated to $\beta \mathrm{C} 1$ [38].

We further validated these results by swapping tags and using a non-conjugable form of NbSUMO1 (NbSU$\left.\mathrm{MO} 1 \Delta^{\mathrm{GG}}\right)$ in the abovementioned assays. During the SUMOylation process, SUMO proteins undergo proteolysis at their C-terminus to expose their di-Glycine motifs. As a result, when we transiently overexpressed $N b S U M O 1 \Delta^{\mathrm{GG}}$ in plants, higher-order conjugation products were absent at the global level suggesting inefficiency of $N b S U M O 1 \Delta^{\mathrm{GG}}$ to undergo SUMOylation (Additional file 1: Fig. S4C). We used GFP-tagged $N b S U M O 1$ and $N b S U M O 1 \Delta^{\mathrm{GG}}$ to validate that the higher-order bands observed are actual conjugated products of NbSUMO1 derived from the SUMOylation cascade. We used MBP-tagged $\beta C 1$ as a substrate for detecting SUMOylation along with GFP-tagged $\mathrm{NbSU}$ $\mathrm{MO} 1$ in conjugable and non-conjugable forms. After CoIP with anti-MBP followed by blotting with anti-GFP and anti-MBP, we observed high molecular weight bands in $\beta C 1$ when co-expressed with a conjugable form of NbSUMO1, but not with non-conjugable form (Fig. 1h).

NbSUMO1 is the only identified SUMO protein in Nicotiana sp., whereas the model plant Arabidopsis has 4 characterized SUMO proteins [39]. To further explore the possibility of interaction between $\beta C 1$ and other SUMO proteins, we used $\mathrm{Y} 2 \mathrm{H}$ assay. As SUMO1 and SUMO2 have highly redundant biochemical functions in Arabidopsis, we used only SUMO1, SUMO3 and SUMO5 of Arabidopsis as prey proteins fused to activation domain (AD) in a yeast two-hybrid screen with $\beta C 1$ fused to the binding domain (BD). We observed a strong interaction of $\beta C 1$ with AtSUMO3 and AtSUMO5 (Additional file 1: Fig. S5A). AtSUMO5 caused auto-activation when fused to $\mathrm{AD}$ domain alone; however, the strength of interaction with $\beta C 1$ in the quadruple (-LWHA) knockout 
media was clearly observed. Auto-activation caused by AtSUMO5 was minimal in -LWHA, but in the presence of $\beta C 1$, the growth of cells was enhanced suggesting interaction. In the case of $A t \mathrm{SUMO} 3$, a strong interaction was observed only with $\beta \mathrm{C} 1$, indicating $\beta C 1$ might also interact with AtSUMO3. To distinguish between SUMOylation and SIM-mediated interactions, we used di-Glycine deleted AtSUMO3 and AtSUMO5. Interestingly, the deletion of the diGlycine motif caused no difference in the interaction of $\beta \mathrm{C} 1$ with AtSUMO5, but completely abolished its interaction with AtSUMO3. These results suggest that other SUMO proteins might also interact with $\beta \mathrm{C} 1$ via SUMOylation or SIM-mediated interactions. In these assays, protein expression and stability of all proteins were verified. All other proteins except AtSUMO5 were expressing at almost equal levels in yeast cells, while AtSUMO5 was expressed at unusually high levels, and might be the reason for its auto-activation (Additional file 1: Fig. S5B) [40]. To further verify the biological significance of the observed interaction between $\beta C 1$ and other SUMO proteins, we overexpressed GFP-tagged versions of both AtSUMO3 and AtSUMO5 along with $\beta \mathrm{C} 1$ and performed an IP with $\beta C 1$ as previously described (Fig. 1f). We observed a very weak pull-down signal of $A t \mathrm{SUMO} 3$ and AtSUMO5 as compared to NbSUMO1 (Additional file 1: Fig. S5C). These experiments suggest that even though $\beta C 1$ is able to interact with other SUMO proteins in yeast, in Nicotiana sp., $\beta C 1$ majorly interacted with NbSUMO1. Together, these results strongly indicate that $\beta C 1$ undergoes SUMOylation in plants and that it interacts with host SUMO proteins.

\section{SUMOylation sites are essential for the stability of $\beta C 1$ in host plants}

Since there are three predicted SUMOylation sites in $\beta C 1$ (Fig. 1a), we explored which among these predicted sites are necessary and sufficient for SUMOylation. We substituted lysine residues to arginine, which will abolish SUMO modification of the consensus sequence without leading to much structural disruption. Since Ss1 (K18) and Ss2 (K24) residues of $\beta C 1$ are close to each other, we designed a double mutant $\mathrm{K} 18,24 \mathrm{R}$ (henceforth $\mathrm{mK} 18,24 \mathrm{R}$ ) to cover both these sites, a single Ss3 (K83) mutant (mK83R) and a null mutant with all three predicted lysines mutated to arginines, i.e. K18R, K24R and K83R (mK18,24,83R) (Fig. 2a). We recombinantly expressed and purified the abovementioned mutants of $\beta C 1$ from $E$. coli and performed an in vitro SUMOylation assay with NbSUMO1. We observed that all predicted lysines (K18, 24 and 83) underwent NbSUMO1 conjugation and mutating these sites to arginine in double mutant or in triple null mutant abolished NbSUMO1 modification in vitro (Fig. 2b). To further confirm the above observations, we also performed an in vitro SUMOylation assay with $\mathrm{NbSUMO} 1$ using short peptides covering the $\beta C 1$ SUMOylation consensus lysine sites. Mutating SUMOylation sites inhibited NbSUMO1 conjugation in vitro (Additional file 1: Fig. S6A). These results indicate that there is a propensity of all 3 predicted sites of $\beta C 1$ to undergo SUMOylation in vitro.

To further understand the importance of these SUMOylation sites of $\beta C 1$, we generated transgenic plants overexpressing SUMOylation site mutants of $\beta C 1$. Along with mK18, 24R, a Ss3 mutant was generated where the consensus lysine K83 was kept intact, while SUMOylation consensus sequence was removed ( $\mathrm{mS} \mathrm{SMs3,} \mathrm{Fig.} \mathrm{2a).} \mathrm{Interestingly,}$ transgenic plants overexpressing mK18, 24R double mutant was devoid of abnormal phenotypes and was similar to GFP overexpressing control plants (Fig. 2c), indicating that SUMOylation in K18, K24 residues of SyYVCV $\beta C 1$ is essential for the development of symptoms in plants. $\mathrm{mS}$ SMs3 mutant overexpressing transgenic plants also showed recovery from severe phenotype to some extent (Fig. $2 \mathrm{~d}$ and Additional file 1: Fig. S6B, C and D).

In order to understand why transgenic plants expressing $\mathrm{mK} 18,24 \mathrm{R}$ double mutant were asymptomatic, we performed detailed molecular analysis. Our immunoblot analysis of $\beta C 1$ and SUMOylation-deficient mutant plants revealed a reduced protein accumulation of mK18, 24R double mutant as compared to WT $\beta C 1$ or other mutants (Fig. 2e). SUMOylation-deficient double mutant mK18, 24R protein accumulated only $10 \%$ of WT $\beta \mathrm{C} 1$ (Fig. 3a and Additional file 1: Fig. S7A). mS SMs3 mutant protein levels were comparable to that of WT $\beta C 1$ (Fig. 2e). The transgenic plants expressing $\beta C 1$ mutant showed disproportionate severity of symptoms, likely indicating a hierarchy in these sites to undergo PTMs and subsequent functions. Reduction in mK18, $24 \mathrm{R}$ protein level was not due to reduced transcription as seen in RT-PCR analysis (Fig. 2e). Further, to verify the observations of transgenic plants, we transiently overexpressed WT $\beta C 1, m K 18,24 \mathrm{R}$ and $\mathrm{mK} 83 \mathrm{R} \mathrm{mu}-$ tants in N. tabacum and performed an IP for $\beta C 1$. Similar to transgenic plants, mK18, 24R mutant levels were significantly reduced in our IP analysis performed from transient overexpression of these proteins (mK18, 24R and mK83R) (Fig. 2f). To understand the SUMOylation status of these three lysines of $\beta \mathrm{C} 1$, we performed a $\mathrm{Co}-$ IP assay by co-expressing MBP-tagged $\beta C 1$ or mK18, 24, $83 \mathrm{R}$ along with GFP-NbSUMO1. We observed a significant decrease in SUMOylation of mK18, 24, 83R triple mutant, indicating that these sites are the major sites of NbSUMO1 conjugation (Fig. 2g). 
A)

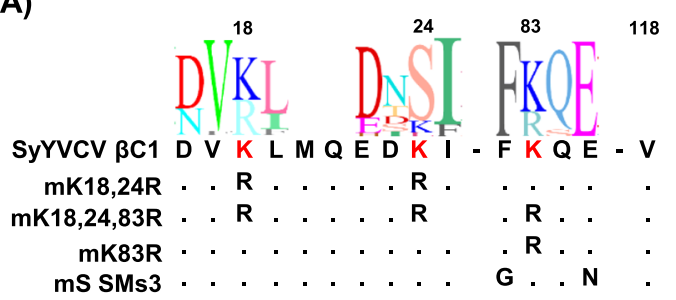

C)
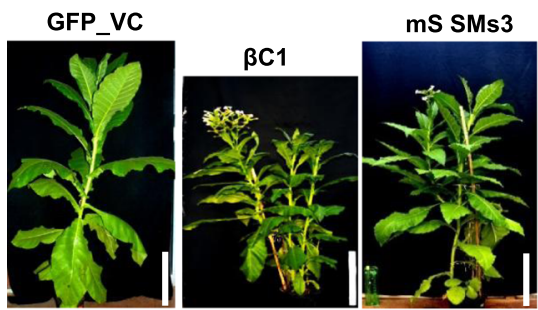

स $5 \propto 8$

E)

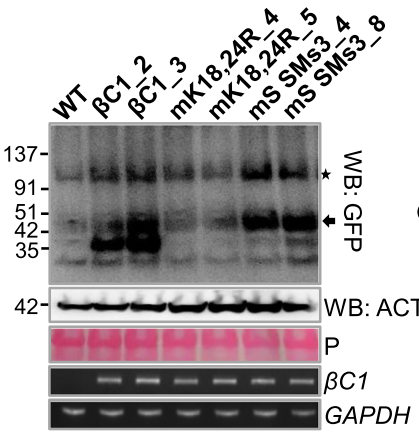

F)

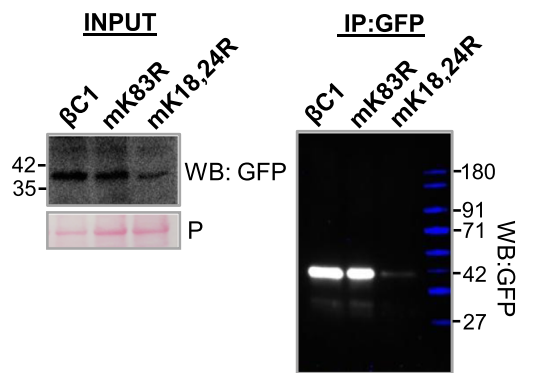

G)

$m K 18,24,83 R$
B)

Mg-ATP + + + - +

$\mathrm{MBP}+-$ - -

$\beta C 1$ - $-\quad++$

$\mathrm{mK} 18,24 \mathrm{R}-\quad+\quad-$

$\mathrm{mK} 18,24,83 \mathrm{R}-\quad+\quad-\quad-$

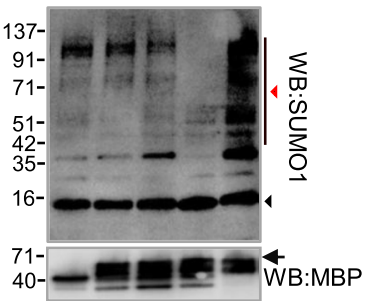

D)

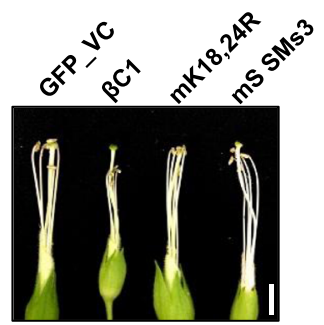

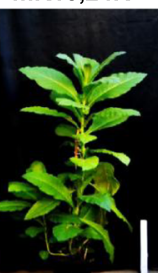

G-NbSUMO1 + + + +

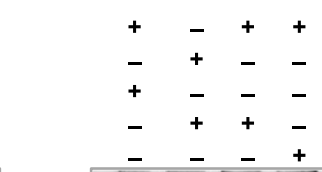

G-NbSUMO1 ${ }^{\triangle \mathrm{GG}}-+$

$\mathrm{MBP}+--$

BC1 -++

$3 \mathrm{R}--+$
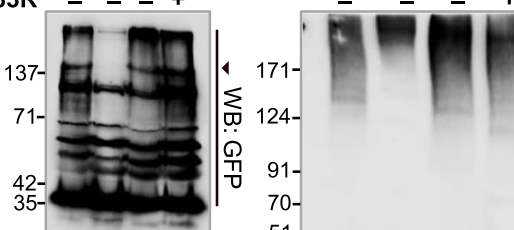

另 91 -
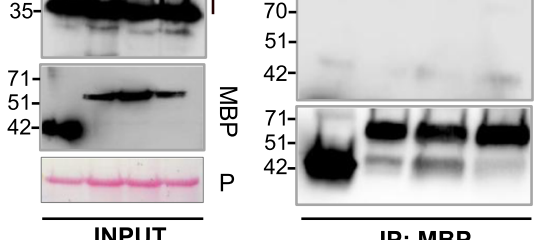

$\sum$

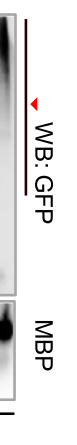

Fig. 2 Mutations in $\beta C 1$ SUMOylation sites abolish pathogenicity. a Design of $\beta C 1$ SUMOylation motif mutants. SeqLogos indicates conservation in amino acid sequences between $\beta C 1$. $\mathbf{b}$ In vitro SUMOylation assay using purified MBP- $\beta C 1$ and its SUMOylation motif mutants. Top blot shows SUMOylation conjugates of $\beta C 1$ probed with anti-SUMO1 and bottom blot is reprobing with anti-MBP. Black arrow indicates intact MBP- $\beta C 1$. Black and red triangles represent NbSUMO1 and poly-SUMOylated substrates, respectively. $\mathbf{c}$, $\mathbf{d}$ Representative phenotypes of GFP- $\beta C 1$ and its SUMOylation motif mutants in transgenic N. tabacum. c Plants. d Flowers without petals. e Western blot to quantify GFP- $\beta C 1$ and different SUMOylation motif mutants in transgenic plants using anti-GFP. The star indicates a non-specific band and the arrow shows GFP- $\beta C 1$. $\mathbf{f} I P$ of GFP$\beta C 1$ and its SUMOylation motif mutants showing stability of the proteins during transient overexpression. Protein ladder overlaid and false colour applied. $\mathbf{g}$ Co-IP of MBP- $\beta C 1$ and its SUMO mutants during co-overexpression of either NbSUMO1 or NbSUMO1 ${ }^{\triangle \mathrm{GG}}$. Black and red triangles indicate NbSUMO1 poly-SUMOylated proteins and NbSUMO1- $\beta C 1$ conjugates, respectively. Protein marker sizes in kDa are indicated. Size bars in c and $\mathbf{d}$ are $36 \mathrm{~cm}(1.2 \mathrm{ft})$ and $0.8 \mathrm{~cm}$ respectively. P, Ponceau staining for total proteins showing RUBISCO

$\mathrm{N}$-terminal SUMOylation-deficient double mutant of $\beta C 1$ is prone to enhanced degradation in plants

It was shown previously that geminiviral $\beta C 1$ from diverse viruses undergo degradation in host cells. TYLCV $\beta C 1$ undergoes ubiquitin-mediated proteasomal degradation, while CLCuMuV $\beta C 1$ directly interacts with ATG8 which leads to an autophagymediated degradation $[13,14]$. To check if mK18, $24 \mathrm{R}$ mutant of $\beta \mathrm{C} 1$ undergoes active degradation in the host, a time course protein analysis of $\beta C 1$ and mK18, 24R mutant was performed (Additional file 1: Fig. S7B). SyYVCV $\beta C 1$ protein levels were slightly 

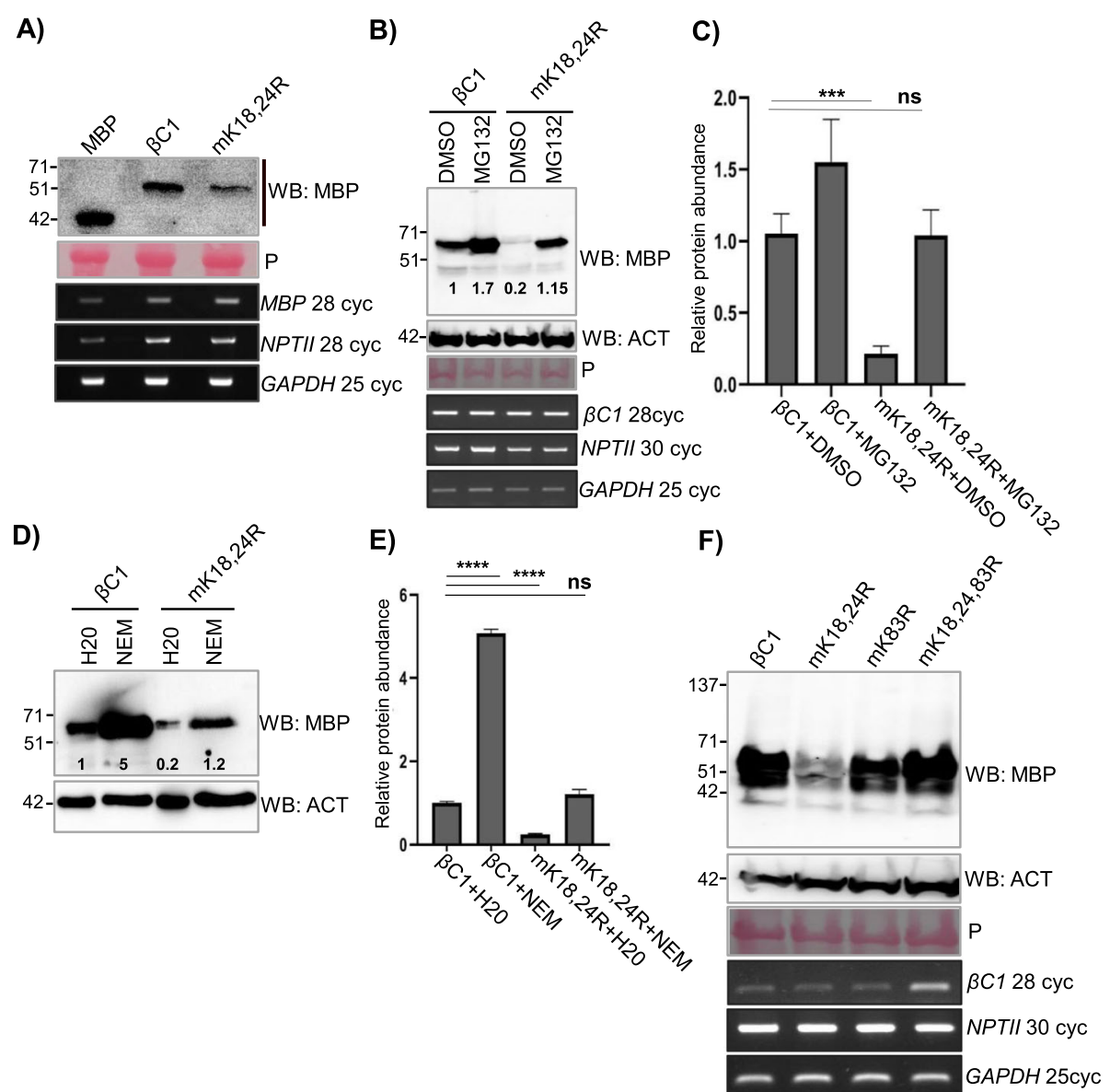

Fig. 3 SUMOylation stabilizes $\beta C 1$ protein in plants. a Stability of transiently overexpressed MBP- $\beta C 1$ and mK18, 24R SUMOylation-deficient double mutant in N. tabacum. Samples were collected 3 DPI. b Accumulation of transiently overexpressed MBP- $\beta C 1$ and mK18, $24 R$ upon MG132 treatment. c Quantification of protein levels after MG132 treatment. $n=3$. d, e Same as $\mathbf{b}$ and $\mathbf{c}$ but with NEM treatment. f Stability of transiently overexpressed MBP-tagged $\beta C 1$ and its SUMOylation motif triple mutant. The number of PCR cycles (cyc) in RT-PCR experiments has been mentioned. Tukey's multiple comparison test with four stars representing $P$ value, $P \leq 0.0001$ and three stars $P \leq 0.001$. $P$, Ponceau staining for total proteins showing RUBISCO large subunit. Other information same as in Fig. 1

reduced after 1 day post infiltration (DPI), whereas mK18, 24R accumulated only to half the level of $\beta C 1$ at 1 DPI and was barely detectable at 2 DPI. To understand the cause of reduced accumulation associated with SUMOylation-deficient mK18, 24R mutant, we employed degradation pathway inhibitors to check if $\mathrm{mK} 18,24 \mathrm{R}$ SUMOylation-deficient mutant undergoes enhanced degradation in vivo. Upon treatment with MG132 (carbobenzoxy-L-leucyl-L-leucyl-L-leucinal), a potent reversible inhibitor of proteasomal activity, protein levels of $\beta C 1$ increased substantially (Fig. 3b), indicating that it undergoes active proteasomal degradation in plants. Interestingly, we also observed a drastic increase in the levels of mK18, 24R mutant whose protein level stabilized more than WT $\beta C 1$ upon MG132 treatment (Fig. 3b and c quantification). We further used a wide spectrum protease inhibitor N-ethylmalemide (NEM) that inhibits cysteine proteases and partially inhibits proteasome [41]. Upon treatment with NEM, mK18, 24R mutant protein levels increased 3- to 5-fold (Fig. 3d and e quantification), suggesting enhanced degradation of $\mathrm{mK} 18,24 \mathrm{R}$ $\beta C 1$ mutant in plants. These assays suggest that the N-terminal SUMOylation-deficient mutant of $\beta C 1$ undergoes enhanced degradation mediated by the host protein degradation pathway.

As observed in protein expression analysis from transgenic plants expressing SUMOylation mutants of $\beta C 1$, there appears to be a disparity between three SUMOylation motifs of $\beta C 1$. To further understand the functional significance of these $\mathrm{N}$ - and C-terminal localized SUMOylation motifs of $\beta C 1$, we performed transient overexpression assays for the abundance of $m K 18,24 R$, $\mathrm{mK} 83 \mathrm{R}$ and $\mathrm{mK} 18,24,83 \mathrm{R} \beta \mathrm{C} 1$ mutants. While the $\mathrm{mK} 18,24 \mathrm{R}$ mutant was unstable in transient assay as well as in transgenic plants, a triple SUMOylation site 
mutant (mK18, 24, 83R) was surprisingly stable (Fig. 3f). To pinpoint the exact SUMOylation motif involved in the stability of $\beta C 1$, we generated individual $\mathrm{K}$ to $\mathrm{R} \mathrm{mu-}$ tants of N-terminal SUMOylation motifs K18 and K24. Interestingly, none of the single mutants was unstable (Additional file 1: Fig. S7C). We speculated that removing all three SUMOylation sites either altered recognition of the protein or other steps in proteasomemediated degradation, leading to the stability of the protein as may be the case of $\mathrm{mK} 18,24,83 \mathrm{R}$. To further confirm that the enhanced degradation of SUMOylation-deficient mK18, 24R mutant in plants is via the plant degradation pathway and not due to intrinsic instability of mutants, we expressed $\beta C 1, \mathrm{mK} 18,24 \mathrm{R}$ and $\mathrm{mK} 18,24,83 \mathrm{R}$ in a yeast WT strain (BY4741). All the mutants were as stable to levels comparable to WT $\beta C 1$ protein (Additional file 1: Fig. S7D). Taken together, all these results confirm that SyYVCV $\beta C 1$ undergoes rapid degradation in plants similar to other viral $\beta C 1$ proteins observed previously. These results also indicate that due to loss of protective marks, mK18, K24R degradation is enhanced suggesting the significance of $\mathrm{N}$ terminal SUMOylation motifs in the stability of $\beta \mathrm{C} 1$.

\section{SUMO-interacting motifs (SIMs) of SyYVCV $\beta C 1$ interact with NbSUMO1}

In multiple proteins that undergo SUMOylation, a complementary stretch of SIM was routinely observed within the candidate protein. In SyYVCV $\beta C 1$, along with three SUMOylation motifs, four SIMs were predicted using SIM prediction softwares JASSA and GPS SUMO. The N-terminal SIM (residue 14-17, SIM1) overlaps with the K18 SUMOylation motif consensus sequence. The second and the third predicted SIM sequences are in an overlapping stretch forming SIM2, 3 (residue 90-93 and 91-94) (Additional file 1: Fig. S8A). The last SIM (SIM4) (residue 101-104) is towards the extreme C-terminal end. It is important to note that SIM2, 3 and SIM4 are in close proximity to the third SUMOylation motif consensus lysine K83.

Plant SUMO proteins are diverse and form a distinct clade even though the SUMO proteins are highly conserved from yeast to mammals (Additional file 1: Fig. S8B). In Arabidopsis, eight SUMO coding genes are known, out of which four SUMO proteins (AtSUMO1, 2, 3 and 5) are known to express and being observed to be functionally active. As reported earlier, we observed differential tissue-specific expression of Arabidopsis SUMO proteins (Additional file 1: Fig. S9A). To identify the SUMO proteins potentially interacting with $\beta C 1$ SIMs, we used NMR titration experiments. We recombinantly expressed and purified ${ }^{15} \mathrm{~N}$-labelled SUMO 1, 2, 3 and 5 from Arabidopsis, and SUMO1 from $N$. benthamiana (Additional file 1: Fig. S9B). Interestingly, AtSUMO3 and 5 were insoluble in E. coli (Additional file 1: Fig. S9C) and, after refolding, exist as soluble higherorder multimers (Additional file 1: Fig. S9G and S9H) whereas NbSUMO1 and AtSUMO1 exist as monomers (Additional file 1: Fig. S9D, E and F). The physiological significance of this multimerization property of plant SUMO proteins is unknown; however, many studies have observed AtSUMO3 and AtSUMO5 localized as nuclear speckles [30]. NbSUMO1 and AtSUMO1 are structurally identical with a pairwise sequence identity of 97\%. We used NbSUMO1 for screening multiple SIMs of SyYVCV $\beta C 1$ through ${ }^{15} \mathrm{~N}$-edited Heteronuclear Single Quantum Coherence (HSQC) experiments using SIM peptides (Additional file 1: Fig. S10A). Upon titration with the SIMs derived from $\beta C 1, N b S U M O 1$ showed interaction with SIM2, 3 and SIM4 (Fig. 4b, c, left panel). However, SIM1 did not show any interaction with NbSUMO1 (Fig. 4a, left panel). Based on the NMR analysis, a structural model of NbSUMO1 indicating the residues involved in interaction with $\beta C 1$ SIMs were predicted (Fig. 4d). The chemical shifts of the backbone ${ }^{1} \mathrm{HN},{ }^{15} \mathrm{~N},{ }^{13} \mathrm{C} \alpha,{ }^{13} \mathrm{C} \beta$ and ${ }^{13} \mathrm{CO}$ resonances of the NbSUMO1 were assigned by standard triple resonance NMR experiments (see the "Methods" section) (Additional file 1: Fig. S11A). The chemical shifts were used in a modelling software CS-ROSETTA [42] to obtain a structural model of NbSUMO1 (Fig. 4d, right panel). The chemical shift perturbations (CSPs) of SIMs were mapped on the NbSUMO1 structure to highlight the SUMO: SIM interface (Fig. 4d, middle panel). To understand the structural interaction and binding pocket involved in NbSUMO1: $\beta$ C1 SIM interaction, we modelled them together based on the CSP data and human SUMO1/IE2-SIM structure (PDB id: 6K5T) in UCSFChimera (Fig. 4d, left panel). IE2 is a human cytomegalovirus protein [43]. The CSPs identified residues 30 to 50 as the region of $N b S U M O 1$ binding $\beta C 1$ SIM 2, 3 motif (Fig. 4b, right panel) and SIM 4motif (Fig. 4c, right panel and Additional file 1: Fig. S11B). To validate these interactions, we further used SIM mutants in our HSQC experiments. As expected, SIM2, 3 or SIM4 mutants did not interact with NbSUMO1 (Additional file 1: Fig. S11C, D and S11E).

We also verified NbSUMO1 interaction with $\beta C 1$ using in vivo and in vitro pull-down assays. $\beta \mathrm{C} 1$ was able to pull-down GFP-tagged NbSUMO1 from plants as well as recombinantly purified $\mathrm{NbSUMO} 1$ in an in vitro pulldown assay. We purified SIM mutated $\beta C 1$ proteins and used them as bait to pull-down NbSUMO1 (recombinantly expressed as 6X HIS NbSUMO1 in E. coli or transiently expressed in plants as GFP-NbSUMO1). As SIM2, 3 and SIM 4 can separately bind to NbSUMO1, mutating both SIM 2, 3 and SIM4 abolished non- 

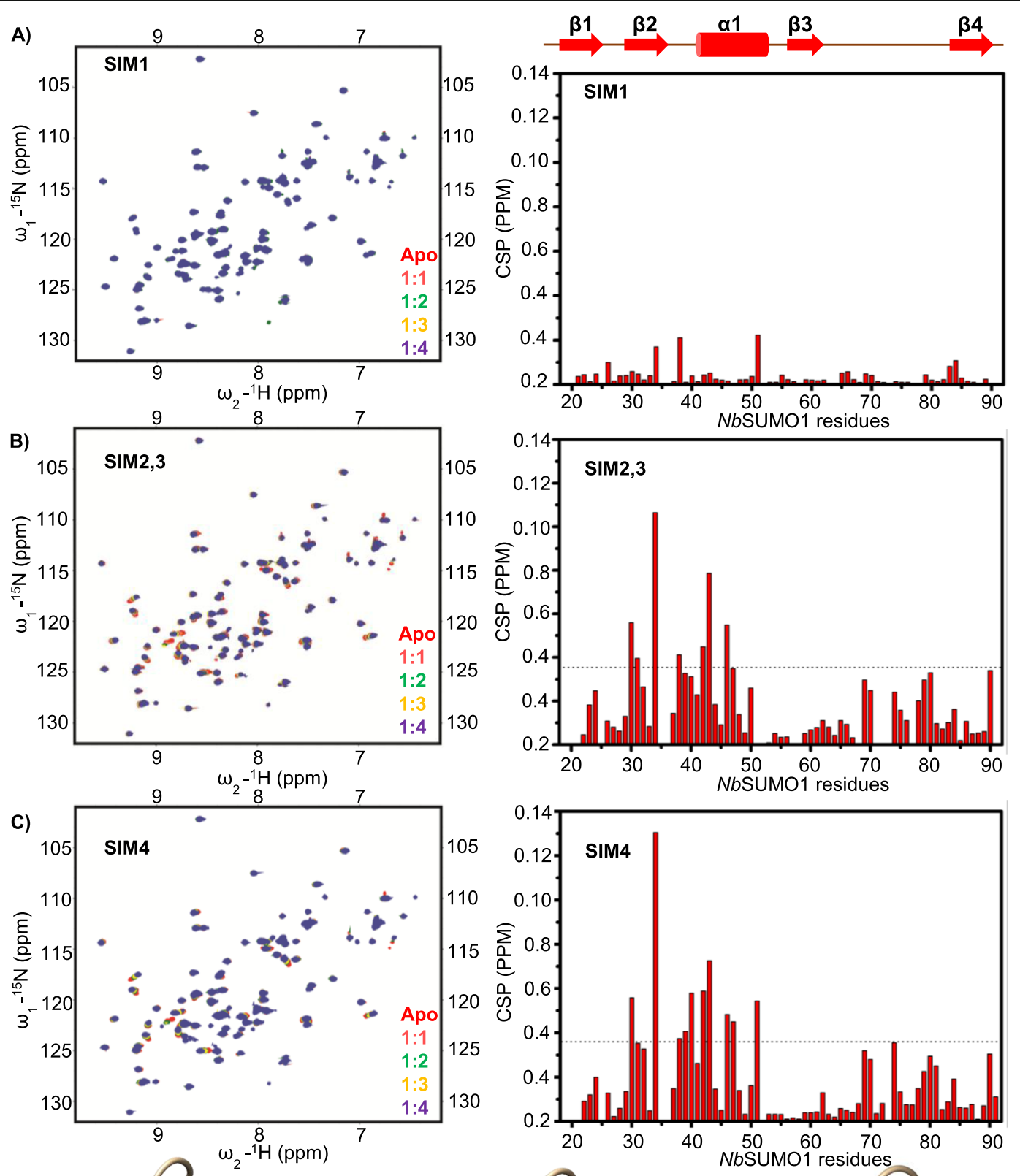

D)
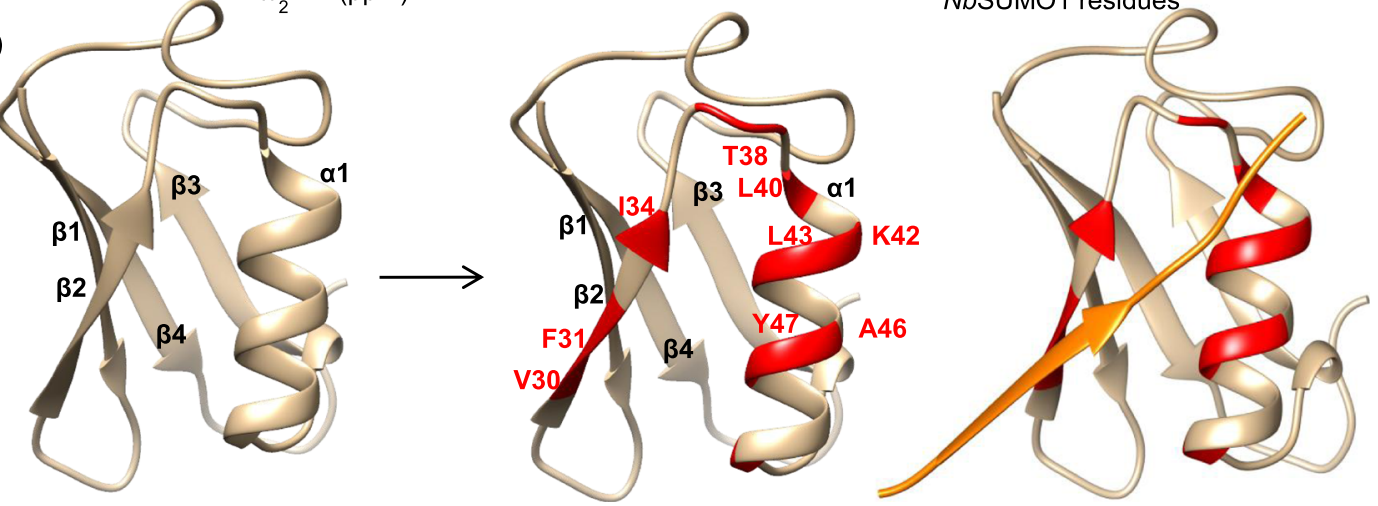

Fig. 4 (See legend on next page.) 
(See figure on previous page.)

Fig. $4 \beta C 1$ SIMs interact with NbSUMO1 as seen with ${ }^{15} \mathrm{~N}-{ }^{1} \mathrm{H}$ HSQC spectrum. a SIM1, b SIM2,3 and c SIM4 (left panel). The right panel shows corresponding CSPS between free and NbSUMO1-SIM-bound form plotted against individual residues of NbSUMO1. The dashed line indicates mean \pm SD of CSP values. Residues above the dash line show binding interface. $\mathbf{d}$ Structure of NbSUMO1. Left panel: NbSUMO1 predicted structure, ribbon and surface representation. Middle panel: residues of NbSUMO1 interacting with BC1 SIM motifs (Red). Left panel: NbSUMO1/ $\beta C 1$-SIM4 model. SIM4 is shown in orange

covalent interactions with SyYVCV $\beta C 1$ (Additional file 1: Fig. S12A and S12B).

\section{SIMs of SyYVCV $\beta C 1$ are essential for its function as a symptom determinant}

SIMs play an important role in protein-protein interactions. To gain further insight into the functional significance of SIMs in $\beta C 1$, we generated $N$. tabacum transgenic lines expressing $\beta C 1$ mutants, where SIMs were mutated to structurally similar motif but without the potential to interact with SUMO (Additional file 1: Fig. S10B). The single mutant of either SIM2, $3(\mathrm{mS}$ SIM2, 3) or SIM4 (mS SIM4) reverted most of the symptoms observed in $\beta C 1$ overexpressing transgenic lines (Fig. 5a). Although single SIM mutants of $\beta \mathrm{C} 1$ transgenic plants had reduced severity of the symptoms when compared to WT $\beta C 1$, they still exhibited mild symptoms such as yellowing of leaves and enhanced branching (Fig. 5b). However, unlike WT $\beta C 1$, transgenic lines stably expressing $\beta C 1$ SIM mutants were fertile, with no defect in floral organs and produced viable seeds (Fig. 5b-d). To understand the basis for this phenotype reversal, we further quantified the level of protein expression of the SIM mutants in transgenic plants. Unlike mK18, 24 R mutant, single SIM mutants were stable in plants and maintained protein levels comparable to that of WT $\beta C 1$ (Fig. 5e). We further validated the stability of SIM mutants by transiently overexpressing Cterminal SIM mutant proteins in plants followed by an IP analysis (Fig. 5f). To reinforce our observation, we transiently overexpressed $\beta C 1$ with its SIM site mutated to structurally similar motif or to an alanine patch completely removing SIM potential (Fig. $5 \mathrm{~g}$ and h quantification) (Additional file 1: Fig. S10B). Surprisingly, SIM mutants were much more stable than WT $\beta C 1$, and mutating a single C-terminal SIM motif was sufficient to increase the stability of the protein considerably. These results suggest that SyYVCV $\beta C 1$ SIM motifs also play an important role in its function and stability.

Since removing SIMs of $\beta C 1$ led to an increase in protein stability, we mutated SIM 2, 3 and SIM 4 of $\beta C 1$ Nterminal SUMOylation motif mutant (mK18, 24R). The $\mathrm{N}$-terminal SUMOylation motif mutant (mK18, 24R) had reduced stability and underwent rapid degradation, whereas upon additional mutations in SIMs enhanced the stability of the protein (Additional file 1: Fig. S12C). We further performed a time course experiment to check for the stability of these SIM and SUMOylation motif mutants of $\beta C 1$. Unlike SUMOylation motif mutant (mK18, 24R), protein levels of SIM mutated $\beta C 1$ were much higher at both 1 and 3 DPI even more than WT $\beta C 1$ levels (Additional file 1: Fig. S12D and 12E quantification).

To understand the reason behind increased stability of SIM mutants, we performed a pull-down experiment using C-terminal SIM mutant (mSIM2, 3, 4) and screened for poly-ubiquitination. WT $\beta C 1$ exhibited poly-ubiquitination as expected and in accordance with previous studies [12, 13, 44]. MBP control showed very little poly-ubiquitination signal. Very interestingly, SIM mutant unlike WT $\beta C 1$ did not accumulate polyubiquitin chains (Additional file 1: Fig. 12F), indicating that disruption of SIM sites is necessary and sufficient to block ubiquitination. Global ubiquitination was not reduced in any of these samples (Additional file 1: Fig. 12F, Input).

\section{SUMOylation motifs and SIMs of SyYVCV $\beta C 1$ are necessary for its function as a viral counter-defence protein}

$\beta C 1$ from multiple begomoviruses have been shown to act as host defence suppressors and mediators of viral replication in host plants. Local viral replication assay was performed to ascertain the role of $\beta \mathrm{C} 1$ in augmenting viral replication. Co-inoculation of infectious SyYVCV DNA-A partial dimer along with p35S: SyYVCV $\beta C 1$ in $N$. tabacum leaves led to enhanced viral accumulation in local leaves (Additional file 1: Fig. S13A). However, mutating SUMOylation motifs of SyYVCV $\beta C 1$ led to the complete abolishment of $\beta C 1$ activity thereby decreasing the viral accumulation (Fig. 6a). Substitution of p35S: SyYVCV $\beta C 1$ with SIM mutants also reduced viral replication in case of SIM2, 3 mutant (Fig. 6b). Similar results were obtained while infecting SyYVCV DNA-A partial dimer on SIM or SUMOylation motif mutant overexpressing transgenic plants (Additional file 1: Fig. S13A and B). We further performed viral replication assay with individual SIM mutants, and as expected, null mutants and structural mutants behaved similarly. Substitution of WT $\beta C 1$ with p35S: mSIM 2, 3, 4 triple mutant in local viral replication assay resulted in the loss of $\beta C 1$ function as a viral replication augmenting protein, resulting in much reduced viral accumulation (Additional file 1: Fig. S13C). 


\section{B) $\mathrm{j}^{\mathrm{C}}$}
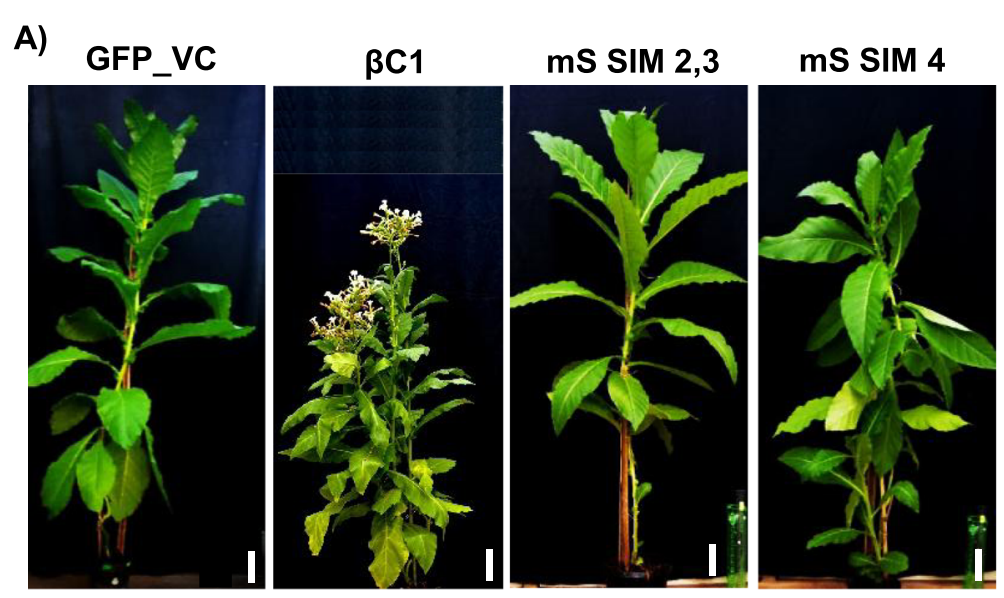

C)

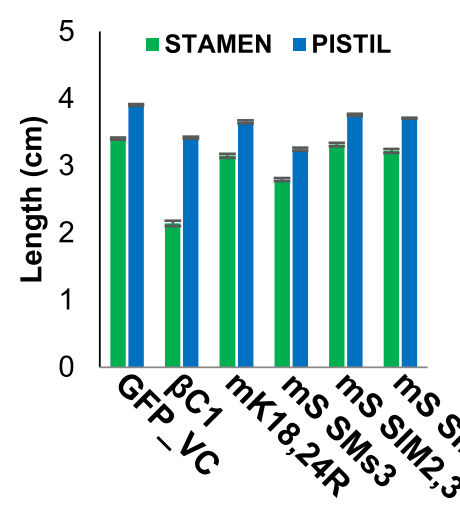

D)

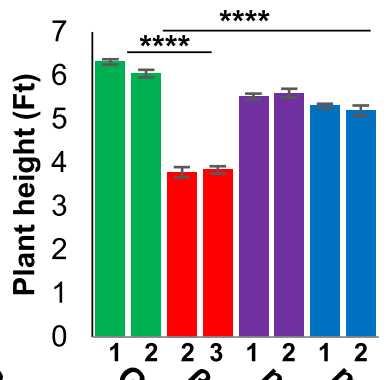

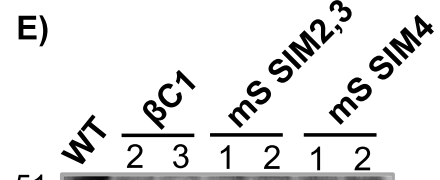

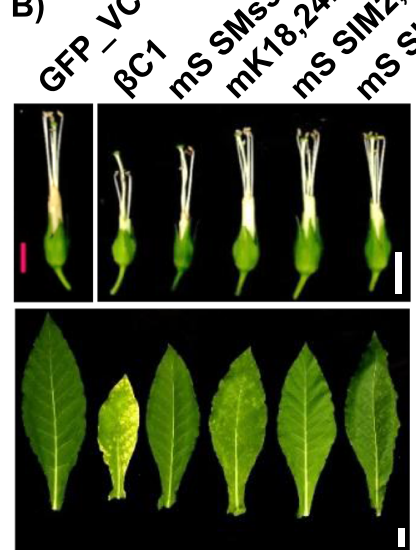

$42-$

42- WB: ACT

$\beta C 1$

$-------G A P D H$
G)

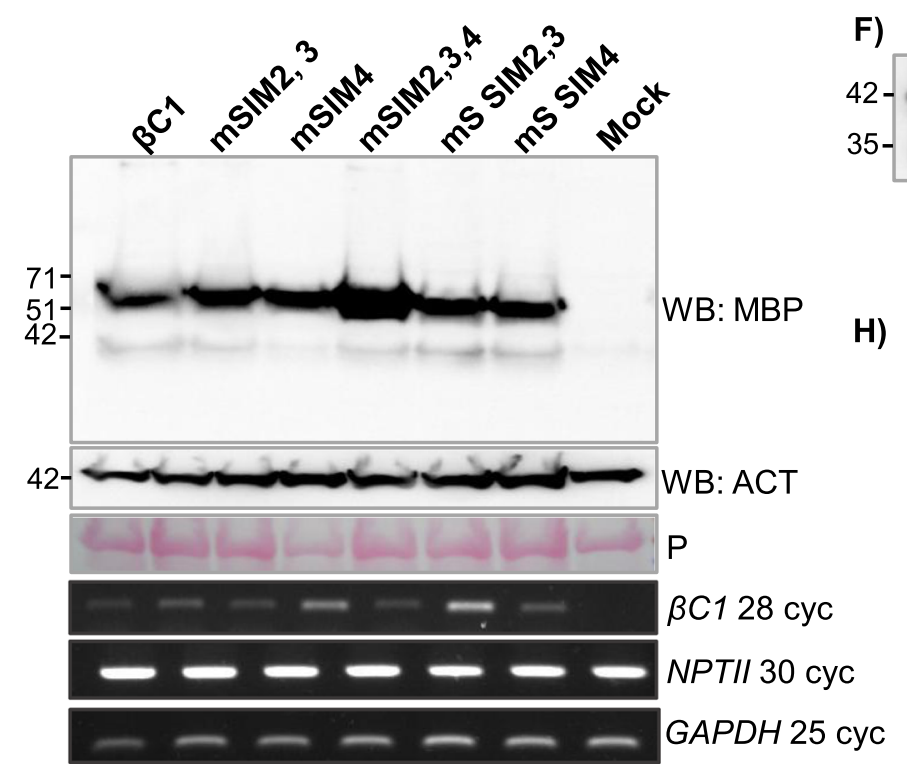

F)
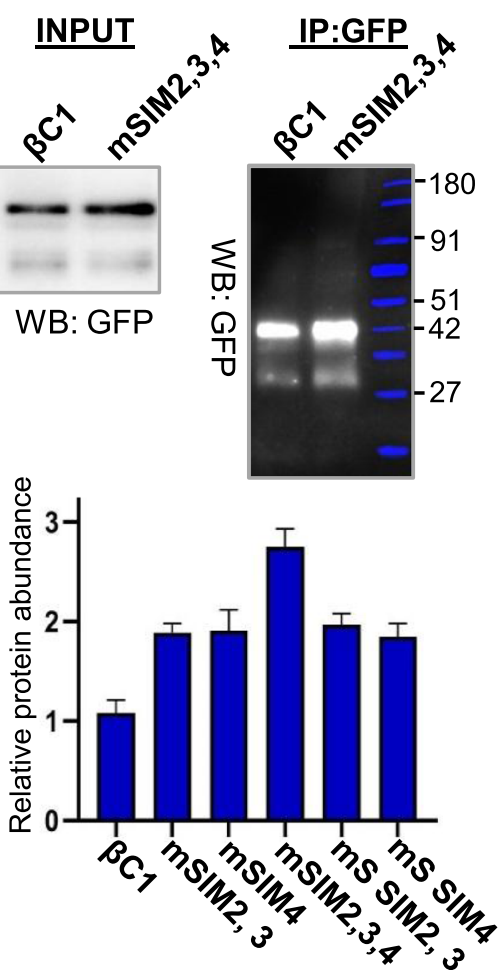

Fig. 5 (See legend on next page.) 
(See figure on previous page.)

Fig. 5 Mutations in SIM abolished infectivity of $\beta C 1$. a-d Habit, flower, leaf and height of transgenic GFP- $\beta C 1$ and its SIM mutants in N. tabacum transgenic lines $(n=3)$. Photographs and measurements (graphs) are presented. e Stability of $\beta C 1$ and SIM mutants overexpressing $N$-terminal GFP-tagged proteins in transgenic plants. Numbers denote individual transgenic lines. $\mathbf{f}$ Stability of SIM mutants during transient overexpression. IP of N-terminal GFP-tagged $\beta C 1$ and mSIM2,3,4 mutants derived from transient overexpression in N. tabacum. $\mathbf{g}$ Western blot showing protein levels during transient overexpression of MBP-tagged $\beta C 1$ and SIM mutants (structural and null mutants) in N. tabacum. $\mathbf{h}$ Quantification of $\mathbf{g}$, the $y$-axis represents protein expression in arbitrary number. $n=3$. Marker sizes in $\mathrm{kDa}$ are indicated. Size bar in $\mathbf{a} 10 \mathrm{~cm}, \mathbf{b}$ top-panel $0.8 \mathrm{~cm}$ and bottom panel $2 \mathrm{~cm}$. P, Ponceau staining for total proteins showing RUBISCO large subunit

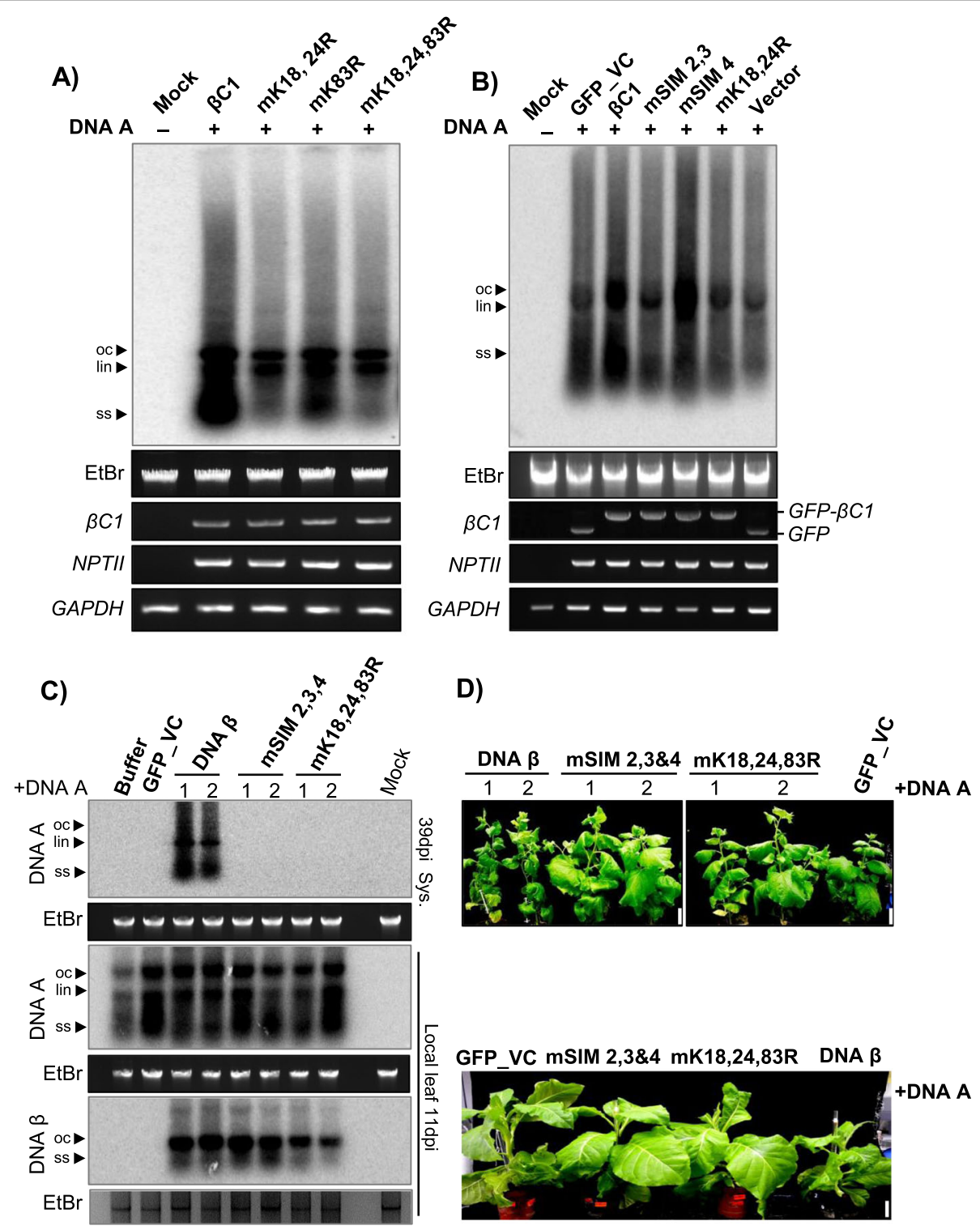

Fig. 6 SUMO/SIM mutants of $\beta C 1$ block viral systemic movement. a Southern blot viral replication assay in local leaves of transgenic plants expressing p35S::GFP-BC1 or its SUMOylation motif mutants. Infectious DNA A clones were used for infection. Total genomic DNA was treated with S1 nuclease before blotting. b Viral replication assay using SIM mutants of GFP- $\beta C 1$. c Southern blot showing viral replication and systemic movement with DNA $\beta$ and SIM/SUMO mutated $\beta C 1$ variants incorporated in DNA $\beta$ in local and systemic leaves of $N$. benthamiana. $\mathbf{d}$ Phenotypes of SyYVCV + DNA $\beta$ or SIM/SUMO mutated $\beta C 1$ incorporated in DNA $\beta$ in N. benthamiana (top) and N. tabacum (bottom). Images were taken at $39 \mathrm{DPI}$. Size bar $5 \mathrm{~cm}$ 
However, surprisingly, when WT $\beta C 1$ was substituted with its SIM4 mutant, we observed enhanced viral accumulation (Fig. 6b). The exact mechanism behind this local enrichment of virus upon mutating $\beta C 1$ SIM 4 is not clear. Most probably, differential functions of the two validated SIMs played a role in this disparity observed in viral replication assay. SIM4 of $\beta C 1$ binds to NbSUMO1 with greater affinity than SIM2, 3 (Fig. 4c, right panel). Even transgenic lines expressing mutated SIM2, 3 or SIM4 motif showed distinct recovery phenotype as compared to $\mathrm{WT} \beta \mathrm{C} 1$. Altogether, these results suggest that $\beta C 1$ SIM and SUMOylation motifs are necessary for its function as a viral pathogenicity determinant protein.

\section{SUMOylation motifs and SIMs of SyYVCV $\beta C 1$ are also essential for systemic viral movement}

In bipartite begomovirus with DNA-A and DNA-B, the $\mathrm{B}$ component codes for $\mathrm{BC} 1$ and $\mathrm{BV} 1$ that act as movement-associated proteins in the systemic spread of the virus. In case of monopartite viruses with a $\beta$ satellite, the function of $\mathrm{BC} 1$ and $\mathrm{BV} 1$ is fulfilled by $\beta$ satellite that codes for a single $\beta C 1$ protein. To verify the function of SyYVCV $\beta C 1$ as a viral movement protein (MP) and to determine the importance of its SIM and SUMOylation motifs in viral movement, we coinoculated partial dimers of SyYVCV DNA-A along with SyYVCV DNA- $\beta$ with its only ORF coding for WT $\beta C 1$ or mSIM 2, 3, 4 (SIM) or mK18, 24, 83R (SUMO) motif mutants incorporated in viral genome, in 3-week-old $N$. benthamiana plants. As replication of DNA $\beta$ is assisted by DNA-A coded Rep protein, we first verified that all DNA $\beta$ dimers are able to replicate locally irrespective of their mutation in $\beta C 1$ SIM or SUMOylation motifs (Fig. 6c). We observed classic viral symptom development (slight yellowing and curling of newly emerging leaves) only in plants inoculated with DNA- $\beta$ coding for WT $\beta C 1$ (Fig. 6d, DNA $\beta$ plants 1 and 2). Upon Southern analysis to verify viral replication in newly emerging systemically infected leaves, we observed the presence of DNA-A replicative form (RF) in the presence of WT $\beta C 1$ containing DNA- $\beta$ (Fig. $6 \mathrm{c}$ ). The symptoms in plants co-inoculated with DNA-A and DNA $\beta$ were much prominent at 39 DPI which was also verified by the increased accumulation of DNA-A in systemic leaves. However, we did not observe detectable levels of DNA-A RFs in plants inoculated with DNA- $\beta$ carrying mutated $\beta C 1$ of either SIM2, 3, 4, or that of SUMOylation motifs such as SUMO K18,24,83R (Fig. 6c, top blot and Additional file 1: Fig. S13D) even at an earlier time point. These results clearly suggest the importance of SIM and SUMOylation motifs of $\beta C 1$ in modulating systemic infection by facilitating viral movement.
SUMOylation of $\beta C 1$ also affects its cellular localization SUMOylation of specific proteins has been implicated in altering the intracellular localization. In order to explore this possibility and to identify mechanism for the perturbations in the functions of $\beta C 1$ upon SIM and SUMOylation motif mutations, we transiently expressed GFPtagged $\beta C 1$ and its mutants in epidermal cells of $N$. benthamiana leaves and analysed their localization using confocal microscopy. WT $\beta \mathrm{C} 1$ was diffusely localized in the nucleus and prominently in the nucleolus matching previous observations for related homologs [45]. Surprisingly, we also observed $\beta C 1$ localizing in the chloroplast, strongly overlapping with chlorophyll auto-fluorescence shown in red (Fig. 7a, 2nd row). Mutating N-terminal double SUMOylation motif mK18, 24R did not result in any qualitative defect in chloroplastic localization (Fig. 7a, 3rd row). However, interestingly, we observed that the Ss3 mutant mK83R showed defects in chloroplastic localization even though its nucleolar localization was unaffected (Fig. 7a, 4th row). The same was observed in the case of triple SUMOylation motif mutant mK18, 24, 83R, where chloroplastic localization was significantly reduced (Fig. 7a, 5th row). We hypothesize that altered localization of $\beta C 1$ mutants might have abolished its ability to support movement and replication of SyYVCV. In contrast, mutating SIM of $\beta C 1$ did not alter the localization. SIM mutants were localized similar to WT $\beta C 1$ in the nucleus, nucleolus and chloroplast, indicating SIM-mediated interactions did not affect localization (Additional file 1: Fig. S14A).

\section{Discussion}

Cells use PTMs as a flexible way of reversibly and effectually controlling protein machinery. PTMs may act as proviral or antiviral machinery, and it has been shown that viruses infecting a range of hosts from yeast to mammals hijack this machinery during infection [46, 47]. Viral proteins might also interact with PTM machinery to regulate both proviral and antiviral effects. Viral proteins also routinely undergo PTMs to modulate infection. Recently, many geminiviral proteins $(\beta C 1, \mathrm{Rep}, \mathrm{AC4}, \mathrm{AV} 1)$ have been shown to undergo PTMs directly [9, 47-49]. We observed SyYVCV $\beta C 1$ has multiple predicted SUMOylation consensus motifs as well as SIMs (Fig. 1a). This was intriguing since no other SyYVCV viral protein, except Rep, has any SIM or SUMOylation motifs. Although $\beta C 1$ from other geminiviruses are modified by phosphorylation and ubiquitination, the functional significance of those PTMs is also not fully understood $[9,13]$. In this report, we identified that SyYVCV $\beta C 1$ gets SUMOylated in host cells to regulate its stability and to mediate interaction with cellular partners. 


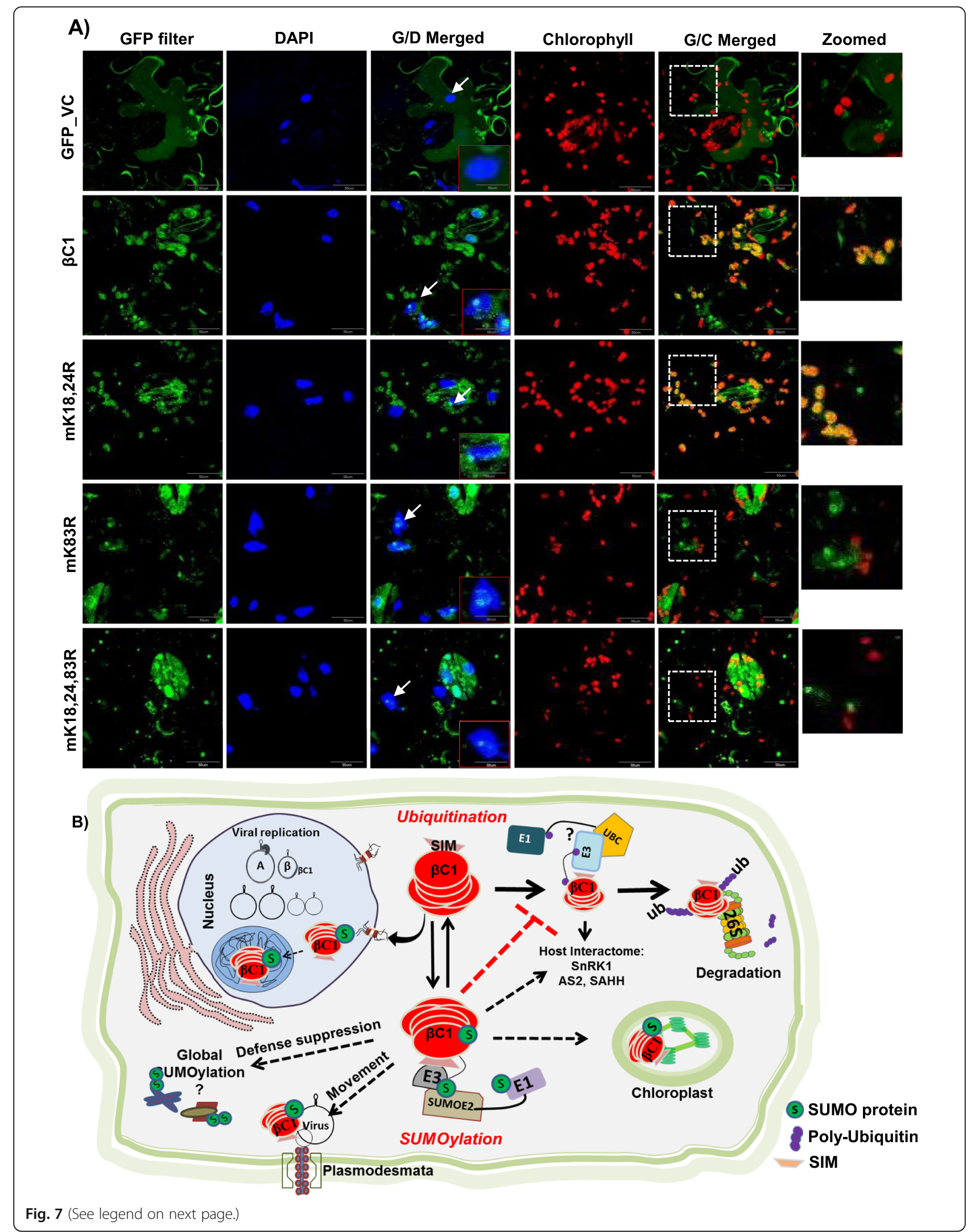


(See figure on previous page.)

Fig. 7 Mutations in SUMOylation motif of $\beta C 1$ alters its localization. a GFP-tagged $\beta C 1$ and its SUMOylation motif mutants were introduced onto $N$. benthamiana leaves as described in the "Methods" section. The nucleus was stained with DAPI, and chlorophyll auto-fluorescence was measured at $650 \mathrm{~nm}$. $\beta C 1$ localization in the nucleus and nucleolus is shown with a white arrow and a representative nucleus was zoomed in the red box. The white box with dashed line represents the zoomed area from G/C merged panel. G/D merged, GFP and DAP filter merged; G/C, GFP and chlorophyll auto-fluorescence merged. Size bar $50 \mu \mathrm{M}$. b Schematic representation showing interplay of multiple PTMs regulating function of $\beta C 1$. Host ubiquitination machinery recognizes $\beta C 1$ and an unknown ubiquitin ligase ubiquitinates $\beta C 1$ via interaction with its SIM motif leading to its degradation. This inhibits viral movement. Viral counter-defence is achieved by interaction of $\beta C 1$ with host SUMOylation machinery. SUMOylated $\beta C 1$ is stable, and this aids in the movement of the virus and pathogenicity

In few $\beta C 1$ sequences, the SUMOylation consensus lysine residue was replaced by structurally similar arginine residue in Ss1 (K18) and Ss3 (K83) sites corresponding to SyYVCV $\beta C 1$. However, since lysine is an absolute requirement for SUMOylation, arginine is unlikely to compensate. Such lysine to arginine replacements are commonly observed among a few viruses infecting tomato (TYLCV, TLCV and ToLCJV), cotton (CLCuV) and chilli (ChiLCV) complexes. Since substitution of lysine with arginine can inhibit SUMOylation even though other amino acids in the vicinity are conserved, this indicates the requirement of SUMOylation as a stronger selection than structural necessity (Fig. 1a and Additional file 1: Fig. S1B). We showed that SyYVCV $\beta C 1$ undergoes SUMO conjugation by NbSUMO1 in plants (Fig. 1g, h). We observed that upon mutating N-terminal SUMOylation site of $\beta C 1$, there was a reduction in stability of $\beta C 1$ (Fig. 2e).

SUMOylation can directly regulate the stability of the protein by inhibiting or increasing access to other PTMs, leading to multiple other functions including protein degradation (SUMO-targeted ubiquitin ligases). SUMOylation can also change the sub-compartmental localization of the protein to cut off access to degradation machinery. $\beta C 1$ from TYLCCNV and CLCuMuV were previously shown to undergo active degradation in cells $[12,13]$. We reasoned that the cause of reduced accumulation of N-terminal SUMOylation-deficient mutant of $\beta C 1$ could be the enhanced degradation by cellular degradation machinery. In agreement with this, upon inhibition of protein degradation pathways, cellular levels of mutant $\beta C 1(\mathrm{mK} 18,24 \mathrm{R})$ reverted to the same level or more than that of WT $\beta C 1$ (Fig. 3b). As expected, this unstable mutant protein did not act as a pathogenicity determinant.

Almost all the proteins having a SUMOylation motif are accompanied by SIMs to play diverse functions in protein-protein interactions [25]. Although precise reasons for propensity of both these modifications in a single protein are still unclear, SIMs are known to help in SUMOylation by increasing the local concentration of SUMO proteins near the SUMOylation site(s). Moreover, SIMs have been shown to interact with SUMOylated proteins by increasing the repertoire of the interaction of a protein without having structural similarity [25]. Among the three SIMs in SyYVCV $\beta C 1$, HSQC NMR analysis identified two SIMs located towards the C-terminus as mediators of interaction with NbSUMO1 (Fig. 4b, c). Mutations in these two SIM motifs led to partial recovery from drastic phenotypes in transgenic plants. Surprisingly, unlike N-terminal SUMOylation motif mutants (mK18, K24R), SIM mutants exhibited enhanced stability of the mutant protein. Also, mutating individual or all SIMs led to increased stability of the protein in cells, suggesting partial redundancy of SIMs in function (Fig. 5g). These results indicate that N-terminal SUMOylation acts as a stability mark, whereas the C-terminal SIM interactions mediate degradation of $\beta C 1$. Multiple observations are in agreement with this hypothesis, for example, mutating SUMOylation motifs removed the protective mark leading to increased degradation. On the other hand, the removal of SIM motifs led to stability. It is likely that SIMs act as marks for host-induced protein degradation that might be mediated by another SUMOylated protein or a protein that binds directly to the SIM patch. Intriguingly, mutating SIMs in N-terminal SUMOylationdeficient mutant background led to increased stability of the otherwise degradation-prone mutant protein (Additional file 1: Fig. S12C).

Interestingly, Haxim et al. [14] also reported a similar observation in $\mathrm{CLCuMuV} \beta \mathrm{C} 1$. Here, V32 residue interacts with ATG8 autophagy mediator leading to $\beta C 1$ degradation, whereas mutating $\mathrm{V} 32$ to $\mathrm{A} 32$ led to hyperactive protein and enhanced symptom induction during infection. In SyYVCV $\beta C 1$, V32 residue has been replaced with a hydrophobic residue F32 that may likely abolish its interaction with ATG8. We hypothesized SyYVCV $\beta C 1$ with only $33 \%$ pairwise sequence similarity with CLCuMuB $\beta C 1$ may follow a similar but divergent strategy when compared to CLCuMuV during infection. Upon mutating SIMs, SyYVCV $\beta C 1$ became ultrastable but lost its function as viral movement protein (Fig. 6c) and pathogenicity (no symptoms observed in transgenic plants expressing SIM mutated $\beta C 1$ when compared to WT $\beta C 1$ ) (Fig. 5a, b). This is in contrast to CLCuMuV $\beta C 1$ where V32 residue was only necessary for protein turnover but not activity. 
The C-terminal SUMOylation motif (K83) is in close proximity to SIM motifs (91 to 94 and 101-104 aa). $\mathrm{Mu}$ tating either SIM motifs or the C-terminal K83 SUMOylation site enhanced the stability of the protein, suggesting a non-redundant but connected function in maintaining stability. Further, we observed that both the SIMs are necessary for function as mutating them led to the loss of symptoms in plants even though the proteins accumulated at optimal levels (Fig. 5a).

In par with our hypothesis, we observed a significant reduction in ubiquitination of C-terminal SIM mutant $\beta C 1$ as compared to WT $\beta C 1$ (Additional file 1: Fig. $\mathrm{S} 12 \mathrm{~F})$. The exact molecular mechanism behind this reduction in ubiquitination is beyond the scope of this work; however, it is highly possible that mutating SIM motifs have deleted a recognition sequence of an unknown ubiquitin ligase, and as a result, it is unable to interact with $\beta C 1$ causing a reduction in ubiquitination. Another possibility is the structural perturbation upon SUMOylation, from the example of PCNA (proliferating cell nuclear antigen), it is clear that both ubiquitination and SUMOylation are required for its function. In PCNA, ubiquitin binding and SUMO binding induced a distinct conformation [50]. It is highly likely that such a mechanism might be regulating $\beta \mathrm{C} 1$ protein, SUMOylation of $\beta C 1$ protein leading to reversible modification of its structure, blocking accessibility of the ubiquitin ligases to $\beta C 1$. Understanding structural perturbation caused by a PTM on a small but complex protein like $\beta C 1$ is difficult due to the nature of the protein that forms heterogeneous soluble higher-order aggregates making it extremely troublesome to resolve even with cryo-EM (data not shown). We also cannot rule out another possibility where $\beta \mathrm{C} 1$ interacts with specific deubiquitinases as observed in HCV NS5A protein [51].

$\beta C 1$ from various viruses have been shown to act as pathogenicity determinants, usually by enhancing viral replication. The function of $\beta \mathrm{C} 1$ as a movement protein was also impaired upon mutating SIM and SUMOylation motifs as observed in our viral replication assays (Fig. 6c). This loss of MP activity was not due to its inefficiency to bind ssDNA (Additional file 1: Fig. S15A). We hypothesize that as SIM and SUMOylation motifs are necessary for cellular interactions, mutations might have led to the loss of activity at the cost of its cellular interactions.

Manipulating SUMOylation pathway can be accepted as an important step in viral infection. Viral proteins modify multiple SUMOylation responsive proteins during infection [17, 52]. Viral manipulation of host SUMOylation machinery might lead to global change in SUMO conjugation as reported with adenovirus GAM1 protein [53] and Epstein-Barr virus (EBV) BRLF1 [54]. None of the plant viral proteins has been shown to have an effect on global SUMOylation. Many plant viral proteins like geminiviral Rep [55] and TuMV NIB [30] have been shown to interact and modify SUMOylation machinery. These viral proteins have specific targets and have not been implicated in global SUMOylation nor do they undergo SUMOylation. Our assays clearly show that SyYVCV $\beta C 1$ induces an increase in global SUMOylation. This modification of global SUMOylation by $\beta \mathrm{C} 1$ is dependent on its SIM and SUMOylation motifs, since mutating either of the motifs compromised $\beta C 1$ global SUMOylation modulating ability observed during both transient and transgenic stable expression of $\beta C 1$ (Additional file 1: Fig. S15B, 15C and 15D). Perturbation in SUMO conjugation by mutating global SUMOylation regulators like SIZ1 (SUMO E3 ligases) or SUMO proteases (EDS4) led to global downregulation or upregulation of SUMO conjugation, respectively, producing early flowering and short stature phenotype in Arabidopsis $[56,57]$. Same was observed in $\beta C 1$ transgenic plants that flowered early and had stunted growth. These findings strongly point towards the manipulation of host SUMO conjugation machinery by $\beta \mathrm{C} 1$ during infection. Our findings that SyYVCV $\beta C 1$ induce SUMO1dependent global SUMOylation suggest that the cellular outcome of $\beta C 1$ protein might be more extensive than is currently known. Further, SUMOylation of $\beta \mathrm{C} 1$ may also play a role in the already known functions of this protein.

We are also able to find additional host signatures altered due to global SUMOylation. It is well known that SUMOylation plays a crucial role in regulating the basal defence of host against pathogens mediated by PR genes [29]. It was shown that in SIZ1 mutant Arabidopsis, PR genes are constitutively expressed suggesting a negative role of SUMO1 conjugation on PR gene regulation. PR genes are in turn regulated by multiple pathways that may be NPR1 dependent or independent [29]. We checked the expression of PR genes during SyYVCV infection and observed that their levels are the same as in uninfected plants. However, very interestingly, we observed that upon mutating SIM or SUMOylation motifs of $\beta C 1$, PR gene expression was upregulated 10-15-fold (Additional file 1: Fig. S16B). Regulators of PR genes such as NPR1, TGA and TCP RNAs were also upregulated only in SIM/SUMO motif mutants of $\beta C 1$ (Additional file 1: Fig. S16A).

In our localization analysis, $\beta C 1$ is localized in the nucleus, nucleolus and chloroplasts. In our analysis, we observed that most of the viruses producing leaf curl symptoms have arginine instead of lysine at the predicted C-terminal SUMOylation motifs of $\beta C 1$ (K83 of SyYVCV $\beta C 1)$. Majority of the yellow vein symptoms producing viruses have lysine at the consensus site 
(Additional file 1: Fig. S17A and17B) (Additional file 2: Table. S2). It is tempting to hypothesize that, in the absence of a chloroplastic transit peptide sequence in $\beta C 1$, K83 consensus site might be the trigger for chloroplastic localization. Localization data available for $\beta C 1$ proteins from the Radish leaf curl virus (RaLCV), TYLCCNV and $\mathrm{CLCuMuB}$ are in agreement with this hypothesis. RaLCV $\beta C 1$ was shown to undergo chloroplastic localization along with nuclear localization, whereas TYLCCNV localized in the nucleus and cell membranes and very weakly in the chloroplast. RaLCV has consensus SUMOylation motif similar to that of $\beta C 1$ (K83, FKQE), whereas TYLCCNB $\beta C 1$ has a modified motif having arginine instead of lysine (R83, FRQE). CLCuMuB $\beta C 1$ lacks a consensus SUMOylation motif at the C-terminal end, and it failed to localize in the chloroplast $[31,45]$. N-terminal SUMOylation motif mutants (mK18, 24R) did not show any drastic defect in localization, but to our surprise, mutating $\mathrm{C}$-terminal SUMOylation motif (K83 as in mK83R or mK18,24,83R) resulted in a clear defect in chloroplastic localization. Interestingly, nuclear and nucleolar localization was not altered in any of the mutants, indicating the major differences might be in chloroplastic localization of the protein (Fig. 7a).

It is unclear why $\beta C 1$ localizes to the chloroplast. RNA viruses prefer chloroplast and peroxisomal membranes as the preferential sites for replication. Geminiviruses mostly replicate in the nucleus but their proteins are known to localize in the chloroplast [58]. It had been previously shown that MP from Potato virus Y $(P V Y)$ and Tobamovirus (ToMV) undergo chloroplastic localization by interacting with Rubisco small subunit $(R b S C)$ [59]. It was observed in these cases that inhibiting interaction between RbSC and MP or inhibiting chloroplastic localization of MP severely inhibited systemic infection of the virus. From our results, it appears that a similar mechanism might be operating in $\beta C 1$, as without a chloroplastic transit peptide, $\beta \mathrm{C} 1$ localizes in the chloroplast since triple SUMOylation motif mutant (mK18, 24, 83R) or single C-terminal (mK83R) mutant that failed to localize in the chloroplast also failed to promote systemic viral infection suggesting a direct link between chloroplastic localization of $\beta \mathrm{C} 1$ and viral systemic infection.

Further, it is not clear if chloroplastic localization is a proviral mechanism or antiviral. It is well known that chloroplast has a cascade of specific proteases and chloroplastic proteins undergo degradation in a complex process. The use of protease inhibitors, especially the cysteine protease inhibitor, increased the protein levels of $\beta \mathrm{C} 1$, suggesting that protease-specific degradation of at least a part of the total pool of viral $\beta \mathrm{C} 1$, thus suggesting localization-specific degradation.
Other PTMs might also add another layer of complexity in the regulation of viral proteins. Phosphorylation has been shown to be one such important PTM regulating the effect of other PTMs [17]. Phosphorylation of the serine adjacent to SIM can lead to differential binding of SUMO protein, as has been shown in the analysis of DAXX protein [60]. Interestingly, SIM4 of $\beta C 1$ has a trailing serine which in our phosphorylation prediction analysis was picked up to be one of the important phosphorylation sites. Further understanding of the multiple PTMs in $\beta C 1$ might offer insights into the defence and counter-defence in DNA virus-host interactions. Since geminiviruses are closely related to human DNA viruses, the results presented here are likely to offer therapeutic insights to develop novel strategies for control of viral diseases across plants and animals.

\section{Conclusion}

Our results elucidated a novel mechanism by which a geminivirus ensures the steady state level of its pathogenicity determinant protein $\beta \mathrm{C} 1$. We show that the virus is able to modulate its host-mediated degradation targeted through its SIM site by robust SUMOylation. This ensures optimal levels of $\beta C 1$ during viral infection, so that a pool of active functional protein is always available. $\beta C 1$ also upregulated global host protein SUMOylation and negatively regulated pathogenicityrelated genes, leading to successful viral infection. These results indicate the presence of another layer of defence and counter-defence operating during plant-virus interactions.

\section{Methods}

\section{Plasmid constructs}

The complete genomic sequence of SyYVCV $\beta C 1$, as well as its amino acid substitution mutants, was cloned from pSD35 harbouring full-length DNA $\beta$ [33]. The pMAL-p5E (New England Biolabs) and pBIN19 vectors were used as templates to amplify MBP and GFP tags to generate fusion constructs. $\beta \mathrm{C} 1$ and its mutants were cloned in pBIN19 using primers having BamHI and SacI sites generating an N-terminal GFP fusion construct driven by $35 \mathrm{~S}$ CaMV promoter. As a control, GFP or MBP alone was also cloned into pBIN19 vector which was used a vector control (pBIN). The substitution mutations in $\beta C 1$ were generated using overlapping PCR primers harbouring the required modification or by sitedirected mutagenesis kit (Invitrogen). For recombinant protein expression in E. coli and their purification, $\beta C 1$ and mutants were cloned in pMAL-p5E using NcoI and NotI sites. Vector was modified by adding a precision protease cleavage site in between $\mathrm{N}$-terminal MBP and $\beta C 1$. For generating infectious clones of DNA $\beta$, partial dimers of DNA $\beta$ having wildtype $\beta C 1$ ORF or mutants 
with specific substitutions were designed and synthesized using Geneart (Thermo Fischer) dsDNA service and the fragments were subcloned into pBIN19 binary vector.

Full-length AtSUMO1, 2, 3 and 5 coding sequences were amplified from cDNA derived from $A$. thaliana young seedlings. NbSUMO1 was amplified from $N$. benthamiana leaves. These amplicons were cloned into expression vector pET-22b (+) (Merck Millipore) carrying N-terminal 6X-HIS tag for protein purification. They were cloned into pBIN19 as fusion proteins either with 3XFLAG or GFP tags translationally fused to the Nterminus of the protein for transient overexpression in plants. Primers used for amplification are listed in Additional file 2: Table S3.

\section{Protein expression and purification}

MBP-tagged SyYVCV $\beta C 1$ and mutants were transformed in Rosetta Gammi DE3 cells (Novagen) according to the manufacturer's directions. For purification, 2-1 culture at OD 0.7 was induced with $0.1 \mathrm{mM}$ IPTG and incubated at $18{ }^{\circ} \mathrm{C}$ for $18 \mathrm{~h}$. Cells were resuspended and lysed in lysis buffer $(25 \mathrm{mM}$ Tris-Cl pH $8,500 \mathrm{mM} \mathrm{NaCl}$, $0.01 \%$ tween 20 , $5 \%$ glycerol, $5 \mathrm{mM} 2$-Mercaptoethanol, $1 \mathrm{mM}$ PMSF, $1 \mathrm{mg} / \mathrm{ml}$ lysozyme and $1 \mathrm{x}$ protease inhibitor cocktail [Roche]) using a sonicator with $5 \mathrm{~s}$ 'ON' and 10 s 'OFF' X 10 cycles at $60 \%$ amplitude in ice. The clarified supernatant was passed through Dextrin Sepharose column (GE Healthcare), and the unbound protein was removed by washing with a buffer $(25 \mathrm{mM}$ Tris- $\mathrm{Cl} \mathrm{pH} 8$, $500 \mathrm{mM} \mathrm{NaCl}, 0.01 \%$ tween 20 and $5 \%$ glycerol). The protein was eluted using $15 \mathrm{mM}$ maltose. Eluted protein was concentrated and subjected to size exclusion chromatography and buffer exchange to Buffer A (25 mM Tris-Cl pH 8, $150 \mathrm{mM} \mathrm{NaCl}$ and 5\% glycerol) using HiLoad 16/600200 pg superdex preparative column (GE).

For purification of SUMO proteins, N-terminally Histagged constructs were transformed in BL21 (DE3) bacterial cells and grown in Luria Bertani (LB) broth. Plant SUMO3 and SUMO5 were insoluble in E. coli unlike their mammalian homologs and were purified by an additional denaturation-refolding step. For NMR experiments, uniformly ${ }^{13} \mathrm{C} /{ }^{15} \mathrm{~N}$-labelled $\mathrm{NbSUMO}$ was purified using a method described previously [61]. The final protein for backbone assignment was obtained in suspension buffer (50 mM Tris $\mathrm{pH} 8.0,100 \mathrm{mM} \mathrm{NaCl}$ and $5 \%$ glycerol). For NMR experiments, the protein sample was supplemented by $10 \% \mathrm{D}_{2} \mathrm{O}$.

\section{Transgenic plants and transient expression}

Transformation of tobacco (N. tabacum, Wisconsin 35) was performed as described previously [62]. Briefly, leaf discs were prepared from 3-week-old N. tabacum plants maintained in tissue culture condition, followed by infection with Agrobacterium strain LBA4404 (pSB1) harbouring gene of interest $[63,64]$. Transformants were selected on kanamycin medium and maintained in greenhouse. For transient overexpression, 3- to 4-weekold N. tabacum leaves were infiltrated using Agrobacterium LBA4404 (pSB1) strains having appropriate genes suspended in an infiltration buffer (10 mM MES, $10 \mathrm{mM}$ $\mathrm{MgCl}_{2}, \mathrm{pH} 5.7$ and $100 \mathrm{uM}$ acetosyringone). A culture of $0.6 \mathrm{OD}$ was incubated for $1 \mathrm{~h}$ before infiltration. For protein expression studies, an equal amount of culture was infiltrated using a needle-less $1-\mathrm{ml}$ syringe onto 2 nd whorl of leaves from the top of either N. tabacum or $N$. benthamiana. During sample collection, equal area of infiltrated leaves was collected and further processed for protein expression analysis.

\section{Plant total protein isolation and western blotting}

Total protein was isolated using the acetone-phenol extraction method [65, 66]. Briefly, $200 \mathrm{mg}$ of tissue was ground in liquid nitrogen and protein was precipitated by $10 \%$ TCA (Trichloroacetic acid, Sigma) in acetone and the resultant precipitate was pelleted by centrifuging at $13000 \mathrm{rpm}$ for $5 \mathrm{~min}$. Pellet was washed with $0.1 \mathrm{M}$ ammonium acetate in $80 \%$ methanol followed by $80 \%$ acetone. The final pellet was further extracted using 1:1 ratio SDS extraction buffer and phenol $(\mathrm{pH} 8.0$ Trissaturated). The resulting mixture was centrifuged at $13000 \mathrm{rpm}$ for $10 \mathrm{~min}$. The protein was precipitated overnight using $0.1 \mathrm{M}$ ammonium acetate in $100 \%$ methanol at $-20^{\circ} \mathrm{C}$. Precipitated protein was pelleted $(13,000 \mathrm{rpm}$ for $30 \mathrm{~min})$ and was washed once with $100 \%$ methanol and then with $80 \%$ acetone, air-dried to remove residual acetone and resuspended in 2X SDS lamelli sample loading buffer containing $6 \mathrm{M}$ urea and $1 \%$ CHAPS.

For western blot analysis, $20 \mu \mathrm{g}$ of total protein was loaded either on a $12 \%$ Tris-Glycine SDS gel or 4-20\% Bio-Rad precast gels. Resolved protein was transferred to nylon membrane (GE (Amersham) Protran, $0.2 \mu \mathrm{m}$ ) and blocked with either 5\% blotting grade blocker (Bio-Rad) or $4 \%$ BSA (Sigma) in TBS with $0.1 \%$ tween 20. Blocked membranes were probed for protein of interest using specific antibody and imaged using Image quant 4000 LAS in chemiluminiscence mode (GE). Blots were stripped using stripping buffer (Restore western stripping buffer, Thermo Fisher) according to the manufacturer's instructions. Bands were quantified and normalized using FIJI. Antibodies used for WB are listed in Additional file 2: Table S4.

\section{Immunoprecipitation}

Infiltrated leaves expressing the protein of interest were powdered in a mortar under liquid $\mathrm{N}_{2}$. About $2 \mathrm{~g}$ of 
tissue was weighed, and to it, 3 volumes of lysis buffer (50 mM Tris-Cl pH 7.4, $150 \mathrm{mM} \mathrm{KCL,} \mathrm{1 \%} \mathrm{Triton} \mathrm{X100,}$ Protease inhibitor $1 \mathrm{X}$ [Roche], NEM 20uM) were added. The supernatant was collected after a spin at $16000 \mathrm{~g}$ for $30 \mathrm{~min}$ and incubated with GFP-Trap (Chromtek) or MBP magnetic beads (NEB) for $3 \mathrm{~h}$ at $4{ }^{\circ} \mathrm{C}$. Beads were magnetically separated from the lysate and washed 5 times in wash buffer (50 mM Tris-Cl, pH 7.4; $150 \mathrm{mM}$ KCL, $1 \mathrm{mM}$ PMSF) until the green colour completely disappeared. The final pull-down beads were transferred to a $1.5-\mathrm{ml}$ tube and again washed twice with wash buffer. The 3X SDS sample dye was added to the beads, and the sample was heated at $70{ }^{\circ} \mathrm{C}$ for $10 \mathrm{~min}$. The pulldown products were resolved in 4-20\% Tris-Glycine SDS gradient gels (Bio-Rad). IP beads used are listed in Additional file 2: Table S4.

\section{In vitro SUMOylation assay}

SUMOylation assay was carried according to a published protocol [61]. For SUMOylation of SyYVCV $\beta C 1$ protein, $150 \mathrm{nM}$ of substrate protein was incubated with $1 \mu \mathrm{M}$ E1 (SAE1/SAE2), $2.5 \mu \mathrm{M}$ E2 (ubc9) and $10 \mu \mathrm{M}$ of 6x-HIS-tagged NbSUMO1-GG. The reaction was initiated by adding $1 \mathrm{mM}$ ATP. The reaction was carried out at room temperature in a buffer containing $25 \mathrm{~mm}$ Tris (pH 8.0), $150 \mathrm{mM} \mathrm{NaCl}, 5 \mathrm{mM} \mathrm{MgCl}_{2}$ and $0.1 \%$ Tween 20. The reaction was terminated by adding $2 \mathrm{X}$ SDS lamelli buffer, followed by boiling at $95^{\circ} \mathrm{C}$ for $1 \mathrm{~min}$ and separating on a $4-20 \%$ gradient gel. For SUMOylation assay using peptides, $5 \mu \mathrm{M}$ of peptide substrate was used. For positive control of the reaction and reconfirmation of SUMOylation of $\beta C 1$, MBP-tagged SyYVCV $\beta C 1$ and SUMO domain mutants of $\beta C 1$ were incubated with SUMOylation cascade enzymes as per the manufacturer's instructions (SUMOylation kit, ENZO life sciences). HsSUMO1 was replaced with NbSUMO1. The reaction was terminated using $2 x$ SDS non-reducing dye and the mixture was resolved in a $4-20 \%$ gradient gel (Bio-Rad), followed by detection with anti AtSUMO1 antibody.

\section{Inhibitor treatment}

Inhibitor treatment was performed as described previously [13]. About $16 \mathrm{~h}$ before collection of leaf samples infiltrated with constructs, $50 \mu \mathrm{M}$ of MG132 (Cellagen) or equal carrier concentration of DMSO (Sigma) were super-infiltrated onto the same leaves. For NEM treatment, $50 \mu \mathrm{M}$ of NEM in infiltration buffer (MES $10 \mathrm{mM}, \mathrm{MgCl}_{2} 10 \mathrm{mM}$ ) were infiltrated $12 \mathrm{~h}$ prior to sample collection. Samples were immediately frozen in liquid $\mathrm{N}_{2}$, and proteins were isolated as mentioned above.

\section{Viral replication assay and southern blotting}

Viral titre assay was performed as previously shown [67, 68]. Partial dimer of SyYVCV DNA-A and 35S: SyYVCV $\beta C 1$ or mutants were mobilized into Agrobacterium strain LBA4404 (pSB1) and co-infiltrated in N. tabacum leaves. Samples were collected 7 DPI. For checking systemic infection, partial dimer of SyYVCV DNA-A and DNA $\beta$ or DNA $\beta$ with mutated $\beta C 1$ were co-infiltrated in 2-week-old $N$. tabacum or N. benthamiana leaves. Genomic DNA from infiltrated and systemic leaves was isolated using the $C T A B$ method [69]. An equal amount of genomic DNA was loaded onto a $0.7 \%$ TNE agarose gel and resolved at $5 \mathrm{~V} /$ $\mathrm{cm}$. The transfer was performed as previously mentioned [70], and blots were probed with full-length DNA-A or DNA- $\beta$, internally labelled with dCTP alpha P32 (BRIT, India) using Rediprime II kit (GE). Blots were scanned using Typhoon Trio Scanner (GE) in phosphorescence mode.

\section{Yeast two-hybrid screening}

All SUMO CDS were translationally fused with the activation domain of pGADT7 AD (Takara Bio). $\beta C 1$ was fused with binding domain and cloned into pGBKT7 BD (Takara Bio). Plasmids were transformed into AH109 strain as described previously [71] Successful transformants were screened on -Leu, -Trp media followed by screening for interaction on -Leu,- Trp, -His with or without 3AT (Sigma-Aldrich). Successful interactions were further screened on -Leu, -Trp, -His, -Ade media.

\section{Synthetic peptides}

All the synthetic peptides for NbSUMO: SIM titration measurements were purchased from Lifetein LLC as lyophilized powders. The peptides were subsequently dissolved in re-suspension buffer $(25 \mathrm{mM}$ Tris $\mathrm{pH} 8,100$ $\mathrm{mM} \mathrm{NaCl}, 5 \%$ glycerol) and used for titration by NMR.

\section{NMR experiments}

All NMR spectra were recorded at $298 \mathrm{~K}$ on an $800-\mathrm{MHz}$ Bruker Avance III HD spectrometer equipped with a cryo-probehead. All spectra were processed with NMRpipe [72] and analysed with NMRFAM-SPARKY [73]. For titrations with SIMs, standard 15N-HSQC were recorded for each protein-ligand concentration. Standard triple resonance $\mathrm{CBCA}(\mathrm{CO}) \mathrm{NH}, \mathrm{HNCACB}, \mathrm{HNCO}$ and $\mathrm{HN}$ (CA) $\mathrm{CO}$ experiments were used from Bruker library for backbone assignments using a $\sim 1 \mathrm{mM}$ uniformly ${ }^{13} \mathrm{C},{ }^{15} \mathrm{~N}$-labelled NbSUMO. Following peak picking of the backbone experimental data in Sparky, the chemical shift lists were submitted to PINE NMR-server [74], and the assigned peak list was verified and completed manually. For the structure of NbSUMO, the above chemical shift lists were submitted to the CS-Rosetta server along with the primary protein sequence. From the obtained structures, the best PDB was reported out of. 


\section{Supplementary information}

Supplementary information accompanies this paper at https://doi.org/10. 1186/s12915-020-00843-y.

Additional file 1: Fig. S1: SyYVCV $\beta C 1$ has multiple conserved SUMOylation sites. A) Table summarizing JASSA prediction of SUMOylation sites in $\beta C 1$. SUMOylation consensus lysine residue is shown in red. PS: represents predictive score. Arrow indicates the direction of the motif. B) Heat map showing conserved lysine and predicted SUMOylation sites among $\beta C 1$ sequences from different viruses. Aligned residue no. indicates the original position of lysine in the protein alignment of $\beta C 1$. SyYCV $\beta C 1$ SUMOylation consensus lysines are highlighted at the top. Fig. S2: SyYVC $\beta C 1$ can interact with NbSUMO1. A) in vitro SUMOylation assay with NbSUMO1-GG and SyYVCV $\beta C 1$ as substrate using purified SUMOylation cascade enzymes. Concentrations of NbSUMO1 are marked. Red and black triangles represents poly-SUMOylated products and free NbSUMO1, respectively. Black arrow indicates E1-NbSUMO1 conjugate. Right triangle on top of the gel indicates timepoints (30 and $60 \mathrm{~min}$ ). B) Yeast two-hybrid assay with binding domain fused $B C 1$ and activation domain fused NbSUMO or NbSUMO1 $\Delta^{\mathrm{GG}}$ (C-terminal di-Glycine deleted, SUMOylation defective), selected in -LW, and screened in -LWH media with $0.2 \mathrm{mM}$ 3AT. Protein marker sizes are indicated in kDa. WB: indicates western blotting using specified antibody. Fig. S3: SyYVCV $\beta C 1$ induces various developmental defects in transgenic plants. A) GFP- $\beta C 1$ expressing transgenic plants showing exerted stigma. Size bar-1 $\mathrm{cm}$. B) Histogram representing stigma and pistil measurements. $N=10$ for each line, two individual lines for GFP- $\beta C 1$ were analyzed. C) Histogram showing height of transgenic GFP$\beta C 1$ plants. Numbers on the $x$-axis indicates transgenic plant line number $N=5 /$ line. D) Graph showing early heading date in GFP- $\beta C 1$ transgenic plants. $N=5 /$ line. Stats: Tukey's multiple comparison test with $P$-value: four, three and two stars representing $P \leq 0.0001,0.001,0.01$, respectively. Fig. S4: SyYVCV $\beta C 1$ undergoes SUMOylation in planta. A) Co-IP of GFP$\beta C 1$ from transgenic plants using anti-GFP antibody followed by WB. Red arrow represents SUMO1 conjugated $\beta C 1$. Black arrow shows unmodified $\beta C 1$. Star shows non-specific band. B) Same as in A) except for WB with anti-AtSUMO1. C) WB of transiently over-expressed GFP tagged NbSUMO1 and $\mathrm{NbSUMO}^{\triangle \mathrm{GG}}$. Red triangle indicates poly-SUMOylated products. Other details are as in Additional file 1: Fig. S2 legend. Fig. S5: SyYVCV BC1 weakly interacts with other plant SUMO proteins. A) Y2H assay showing interaction of $\beta C 1$ with plant SUMO proteins. Deletion of diGlycine motif of SUMO proteins abolishes SUMOylation. Top triangle represents dilutions. B) WB analysis of $Y 2 \mathrm{H}$ transformants. $A D$ and $B D$ domain-fused proteins were detected with anti-HA and anti-MYC antibodies, respectively. Black arrow indicates BD fused $\beta C 1$. Arrow head indicates AD fused plant SUMO proteins. C) Co-IP of $\beta C 1$ with eGFP tagged NbSUMO1, AtSUMO3 and AtSUMO5. $\beta C 1$ and SUMO proteins were COexpressed transiently in N. tabacum. $\beta C 1$ was immuno-precipitated and checked for the presence of conjugated SUMO proteins. About 4-20\% denaturing reducing gel was used. The blot was first probed with antiGFP followed by anti-MBP. Other details are as in Additional file 1: Fig. S2 and S4 legends. Fig. S6: SUMOylation of $\beta C 1$ is required for symptom development. A) Top-panel: in-vitro SUMOylation assay using purified SUMO conjugation components and HA tagged $\beta C 1$ peptides. Lower panel: sequences of peptides. Red and black triangles represent polySUMOylated and mono-SUMOylated $\beta C 1$ peptides, respectively. B) Graph showing initiation of flowering in $\beta C 1$ and SUMO mutant-expressing plants. C) Histogram representing height of transgenic GFP- $\beta C 1$ and $\beta C 1$ SUMOylation mutant plants. D) Graphs showing stamen and pistil measurements in $\beta C 1$ and its SUMOylation motif mutant plants. Other details are as in Additional file 1: Fig. S2 legend. Fig. S7: SUMOylation is required for the stability of $\beta C 1$ in planta. A) WB analysis to check stability of transiently over-expressed MBP tagged $\beta C 1$ and $\mathrm{mK} 18,24 \mathrm{R}$ mutant in N. tabacum. Varying concentrations of protein was loaded. Samples were collected at $4 \mathrm{dpi}$. B) Transient protein expression followed by time course analysis of protein levels for MBP- $\beta C 1$ and its mutant. C) Transient expression and WB of individual SUMOylation site lysine mutants of MBP$\beta C 1$. Samples were collected at $4 \mathrm{dpi}$. D) Expression of HA tagged $\beta C 1$ and its SIM, SUMOylation motif mutants in WT yeast (BY4741). Black arrow represents $\mathrm{MBP}-\beta C 1$ protein $(59 \mathrm{kDa})$, arrow head represents broken MBP and star represents non-specific band. P: Ponceau staining for total proteins showing RUBISCO. Other details are as in Additional file 1: Fig. S2 legend. Fig. S8: SIM sites in SyYVCV $\beta C 1$ and phylogeny of its potential partner SUMO proteins. A) Table showing SIM site prediction in $\beta C 1$ using JASSA software. Predicted SIM residues are highlighted in blue. P.S. indicates predictive score. B) Phylogenetic tree of SUMO proteins. Tree was built in MEGA software using Maximum Likelihood method with 100 bootstraps. Fig. S9: Expression analysis and purification of plant SUMO proteins. A) Relative expression of Arabidopsis SUMO RNAs in various plant tissues of $A$. thaliana. B) CBB stained gels showing purified ${ }^{15} \mathrm{~N}$ labelled recombinant 6 X HIS tagged SUMO proteins. C) WB with anti-HIS confirming the presence of SUMO5 in pellet. $\mathrm{P}$ and $\mathrm{S}$ represent pellet and supernatant, respectively. D-H) Size exclusion profile of different recombinantly NiNTA purified SUMO proteins on a SD75 column. Void volume $40.25 \mathrm{ml}$. Additional details are as in Additional file 1: Fig. S2 legend. Fig. S10: Sequence of SIM mutants. A) Sequence of SIM and mutated SIM peptides used for HSQC experiment. B) Table showing predicted SyYVCV $\beta C 1$ SIM binding sites and various structural and null mutants created in this study. mSIM1, mSIM2,3, mSIM4 and mSIM2,3,4 are null mutants, whereas $\mathrm{mS} \mathrm{SIM2,3}$ and $\mathrm{mS}$ SIM4 are structural mutants.

Fig. S11: SIM sites in $\beta C 1$ C-terminal end interact with NbSUMO1. A) The ${ }^{15} \mathrm{~N}-{ }^{1} \mathrm{H}$ edited HSQC spectrum of NbSUMO1. The backbone amide assignments are labeled beside the peaks. The glutamine and asparagine side chains are connected by black horizontal lines. B) Zoomed HSQC spectrum of NbSUMO1 showing chemical shift in 3 residues (A46, T38 and L43) upon titration with SIM2,3 indicating interaction. Q71, L76 did not show any shift. C), D), E) The ${ }^{15} \mathrm{~N}-{ }^{1} \mathrm{H}$ HSQC spectrum of NbSUMO1 with different titrations involving $\beta C 1$ mutated SIMs. C) pmSIM1, D) pmSIM2,3 and E) pmSIM4. No significant interactions were observed during HSQC titrations. Fig. S12: SIM sites in SyYVCV $\beta C 1$ regulate its stability. A) Semi-in vivo pulldown assay using purified MBP tagged $\beta C 1$ and SIM mutants. These were used to pull down GFP tagged NbSUMO1 ${ }^{\Delta \mathrm{GG}}$ from $N b S U M O 1^{\triangle G G}$ over-expressing plant lysate. B) in vitro pull down of 6X-HIS tagged NbSUMO1 using MBP affinity purification by co-incubation with MBP- $\beta C 1$ or its SIM mutants. C) Transient over-expression of MBP tagged $\beta C 1, m K 18,24 R$ and mK18,24R mSIM2,3 mutant in $N$. tabacum followed by WB with anti-MBP. D, E) Graph and representative blot showing transient protein expression followed by time point analysis of protein level of MBP- $\beta C 1$ and its SUMOylation and SIM mutants. F) WB for pull-down product of MBP-BC1, MBP and MBP tagged C-terminal SIM mutant with anti-Ubiquitin detecting poly-ubiquitin. Purple triangle in F) indicates poly-Ub. Additional details are as in Additional file 1: Fig. S2 legend. Fig. S13: SIM and SUMOylation motifs of $\beta C 1$ are necessary for its pathogenicity determinant function. A) Southern blot showing viral replication in transgenic plants. Plants were infected using partial dimer of DNA-A on GFP- $\beta C 1$ or its SUMOylation motif mutant expressing transgenic plants. About $10 \mu \mathrm{g}$ of genomic DNA was treated with $\mathrm{S} 1$ nuclease and was loaded in a TNE gel for Southern blotting. B) Same as A) but using SIM mutant plants. Right and left panel represents S1 untreated and treated samples, respectively. C) Southern blot showing viral replication assay. Infection was performed with partial dimer of SyYVCV DNA-A along with over-expression of p35S:: GFP- $\beta$ C1 or individual $\beta$ C1 SIM motif mutants. About $4 \mu \mathrm{g}$ of genomic DNA was loaded for blotting. D) Systemic infection assay of SyYVCV DNA-A with WT DNA- $\beta$ and SIM/SUMO mutated $\beta C 1$ variants incorporated in DNA- $\beta$, in local and systemic leaves of $N$. benthamiana at $21 \mathrm{dpi}$. All samples except D) were collected at 11 dpi for local infection. All blots were probed with full length DNA-A probe. Fig. S14: SUMOylation motif in $\beta C 1$ is important for its subcellular localization. A) Localization of GFP tagged $\beta C 1$. All listed proteins were transiently over-expressed in $N$. benthamiana leaf epidermis. Nucleus was stained with DAPI and chlorophyll autofluroscence was measured at 650 $\mathrm{nm}$. $\mathrm{BC} 1$ localization in nucleus and nucleolus has been shown with white arrow and a representative nucleus has been zoomed in red box. White box with dashed line represents the zoomed in area from G/C merged panel. (G/D merged) GFP and DAPI filter merged; (G/C) GFP and chlorophyll autofluroscence merged. Vector GFP and $\beta C 1$ panels were taken for comparison and are same as in Fig. 7. Fig. S15: SyYVCV $\beta C 1$ induces global SUMOylation. A) Gel shift assay to check for binding of $\beta C 1$ or SIM and SUMOylation motif mutants with ssDNA (49 nt). Protein was incubated in increasing concentrations with probe. $(+)$ and $(++)$ 
represents 2 and $5 \mu \mathrm{g}$ of protein, respectively. B) Global SUMOylation induced by $\beta C 1$. Total protein was extracted from GFP- $\beta C 1$ expressing transgenic plants and assessed for NbSUMO1 directed global SUMOylation using anti-AtSUMO1. C) MBP-BC1 or its SIM/SUMO motif mutants were transiently over-expressed in N. tabacum and total protein was extracted at 3 dpi. Followed by a WB analysis with anti-AtSUMO1 antibody to detect NbSUMO1 directed global SUMOylation. D) Same as C) except single SIM mutants of MBP- $\beta C 1$ were used along with double C-terminal SIM mutants. P indicates ponceau staining . Fig. S16: SIM and SUMOylation motif of $\beta C 1$ is essential for host defense suppression. Expression profile of host defense genes in systemic leaves of plants infected with DNA A $+\beta$ or DNA A $+\beta$ with $\beta C 1$ mutated in SIM $(m S I M 2,3,4)$ or SUMO motifs (mK18,24,83R). A) Expression fold difference of various defense regulator genes. B) Expression fold difference of pathogenesis related genes PR1 and PR5. $R$ at the end of sample labels indicates biological replicate. Different letters above each bar indicate significant difference (ANOVA, Tukey-Kramer test, $p<0.05$ ). Fig. S17: Conservation of SUMOylation sites among viruses producing similar symptoms. A) Seqlogo of begomoviral $\beta C 1$ sequences mostly associated with leaf curl symptoms. B) $B C 1$ sequences derived from viruses associated with leaf yellowing/ mosaic symptoms. Fig. S18: Uncropped images of blots in main Fig. 1, 2, 3, 4, 5, 6, 7 and replicates of western blots. Fig. S19: Uncropped images of blots in Fig. S1-16.

Additional file 2: Table S1: Accession numbers used for building alignments. Table S2: Accession numbers used for K83 site analysis. Table S3: List of primers used in this study. Table S4: List of antibodies and materials used for IP.

\section{Acknowledgements}

We thank members of Shivaprasad lab and Prof. K. Veluthambi for suggestions. We thank the Next Generation Genomics, radiation, mass-spec, NMR and Central imaging facilities (CIFF) at NCBS-TIFR, Bangalore. We thank Prof. Deepak Nair and his lab for modified pET 22b+ vector and suggestions. We thank Prof. Patrick D'Silva for the By4741 yeast strain and Prof. Utpal Nath for the AH109 strain. We thank Dr. Soumita Das for help with DNA A partial dimer cloning

\section{Authors' contributions}

P.V.S. designed the study and provided resources and funding. A.N. performed and analysed almost all the experiments and analysed and compiled data. K.S.C. and R.D. performed NMR experiments. V.J. purified SUMO proteins. P.V.S. and A.N. wrote the manuscript. The authors read and approved the final manuscript.

\section{Funding}

This work was supported by NCBS-TIFR core funding and grants (BT/ PR12394/AGIII/103/891/2014;BT/IN/Swiss/47/JGK/2018-19; BT/PR25767/GET/ 119/151/2017) from the Department of Biotechnology, Government of India. P.V.S. is a recipient of the Ramanujan Fellowship (SR/S2/RJN-109/2012; Department of Science and Technology, Government of India). R. D lab is funded through Ramalingaswamy fellowship (BT/HRD/23/02/2006) and NCBS-TIFR core grants.

\section{Availability of data and materials}

All data generated or analysed during this study are included in this published article (and its supplementary information files). Assigned chemical shift list of NbSUMO1 has been submitted to BMRB (Biological Magnetic Resonance Bank) [75]. BMRB entry accession number: '50142'. (http://www. bmrb.wisc.edu/data_library/summary/index.php?bmrbld=50142).

\section{Ethics approval and consent to participate}

Not applicable.

\section{Competing interests}

The authors declare no conflicts of interest.

\section{Author details}

${ }^{1}$ National Centre for Biological Sciences, Tata Institute of Fundamental Research, GKVK Campus, Bellary Road, Bangalore 560065, India. ${ }^{2}$ SASTRA University, Thirumalaisamudram, Thanjavur 613401, India. ${ }^{3}$ Present address:
BIOSS Centre for Biological Signalling Studies, Faculty of Biology, Albert-Ludwigs-Universitaet Freiburg, 79104 Freiburg im Breisgau, Germany.

Received: 8 June 2020 Accepted: 9 August 2020

Published online: 31 August 2020

\section{References}

1. Hanley-Bowdoin L, Bejarano ER, Robertson D, Mansoor S. Geminiviruses: masters at redirecting and reprogramming plant processes. Nat Rev Microbiol. 2013;11:777-88. https://doi.org/10.1038/nrmicro3117.

2. Heyraud-nitschke F, Schumacher S, Laufs J, Schaefer S, Schell J, Gronenborn B. Determination of the origin cleavage and joining domain of geminivirus rep proteins. Nucleic Acids Res. 1995;23:910-6.

3. Muñoz-Martín A, Collin S, Herreros E, Mullineaux PM, Fernández-Lobato M, Fenoll C. Regulation of MSV and WDV virion-sense promoters by WDV nonstructural proteins: a role for their retinoblastoma protein-binding motifs. Virology. 2003;306:313-23. https://doi.org/10.1016/500426822(02)00072-7.

4. Trinks D, Rajeswaran R, Shivaprasad PV, Akbergenov R, Oakeley EJ, Veluthambi K, et al. Suppression of RNA silencing by a geminivirus nuclear protein, AC2, correlates with transactivation of host genes. J Virol. 2005;79: 2517-27. https://doi.org/10.1128/JVI.79.4.2517-2527.2005.

5. Stanley J, Latham JR. A symptom variant of beet curly top geminivirus produced by mutation of open reading frame C4. Virology. 1992;190:506-9. https://doi.org/10.1016/0042-6822(92)91243-N

6. Stanley J, Latham JR, Pinner MS, Bedford I, Markham PG. Mutational analysis of the monopartite geminivirus beet curly top virus. Virology. 1992;191:396405. https://doi.org/10.1016/0042-6822(92)90201-Y.

7. Ribet D, Cossart P. Post-translational modifications in host cells during bacterial infection. FEBS Lett. 2010;584:2748-58. https://doi.org/10.1016/j. febslet.2010.05.012.

8. Jakubiec A, Tournier V, Drugeon G, Pflieger S, Camborde L, Vinh J, et al. Phosphorylation of viral RNA-dependent RNA polymerase and its role in replication of a plus-strand RNA virus. J Biol Chem. 2006;281:21236-49. https://doi.org/10.1074/jbc.M600052200.

9. Shen Q, Liu Z, Song F, Xie Q, Hanley-Bowdoin L, Zhou X. Tomato S1SnRK1 protein interacts with and phosphorylates $\beta C 1$, a pathogenesis protein encoded by a geminivirus $\beta$-satellite. Plant Physiol. 2011;157:1394-406. https://doi.org/10.1104/pp.111.184648.

10. Deom CM, Lapidot M, Beachy RN. Plant virus movement proteins. Cell. 1992; 69:221-4. https://doi.org/10.1016/0092-8674(92)90403-Y.

11. Reichel C, Beachy RN. Degradation of tobacco mosaic virus movement protein by the 265 proteasome. J Virol. 2000;74:3330-7. https://doi.org/10. 1128/JVI.74.7.3330-3337.2000

12. Jia Q, Liu N, Xie K, Dai Y, Han S, Zhao X, et al. CLCuMuB BC1 subverts ubiquitination by interacting with NbSKP1s to enhance geminivirus infection in Nicotiana benthamiana. PLoS Pathog. 2016;12:e1005668. https:// doi.org/10.1371/journal.ppat.1005668.

13. Shen $\mathrm{Q}, \mathrm{Hu} T$, Bao M, Cao L, Zhang H, Song F, et al. Tobacco RING E3 ligase NtRFP1 mediates ubiquitination and proteasomal degradation of a geminivirus-encoded BC1. Mol Plant. 2016;9:911-25.

14. Haxim Y, Ismayil A, Jia Q, Wang Y, Zheng X, Chen T, et al. Autophagy functions as an antiviral mechanism against geminiviruses in plants. Elife. 2017:6. https://doi.org/10.7554/eLife.23897.

15. Mei $Y$, Wang Y, Hu T, Yang X, Lozano-Duran R, Sunter G, et al. Nucleocytoplasmic shuttling of geminivirus $C 4$ protein mediated by phosphorylation and myristoylation is critical for viral pathogenicity. Mol Plant. 2018;11:1466-81. https://doi.org/10.1016/j.molp.2018.10.004.

16. Florentino LH, Santos AA, Fontenelle MR, Pinheiro GL, Zerbini FM, BaracatPereira MC, et al. A PERK-like receptor kinase interacts with the geminivirus nuclear shuttle protein and potentiates viral infection. J Virol. 2006;80:664856. https://doi.org/10.1128/JVI.00173-06

17. Saleh A, Withers J, Mohan R, Marqués J, Gu Y, Yan S, et al. Posttranslational modifications of the master transcriptional regulator NPR1 enable dynamic but tight control of plant immune responses. Cell Host Microbe. 2015;18: 169-82. https://doi.org/10.1016/j.chom.2015.07.005.

18. Chau V, Tobias J, Bachmair A, Marriott D, Ecker D, Gonda D, et al. A multiubiquitin chain is confined to specific lysine in a targeted short-lived protein. Science (80- ). 1989:243:1576-83. https://doi.org/10.1126/science. 2538923. 
19. Deng L, Wang C, Spencer E, Yang L, Braun A, You J, et al. Activation of the IKB kinase complex by TRAF6 requires a dimeric ubiquitin-conjugating enzyme complex and a unique polyubiquitin chain. Cell. 2000;103:351-61. https://doi.org/10.1016/S0092-8674(00)00126-4

20. Johnson LN, Barford D. The effects of phosphorylation on the structure and function of proteins. Annu Rev Biophys Biomol Struct. 1993;22:199-232. https://doi.org/10.1146/annurev.bb.22.060193.001215.

21. Park HJ, Kim W-Y, Park HC, Lee SY, Bohnert HJ, Yun D-J. SUMO and SUMOylation in plants. Mol Cells. 2011;32:305-16. https://doi.org/10.1007/ s10059-011-0122-7.

22. Nayak A, Müller S. SUMO-specific proteases/isopeptidases: SENPS and beyond. Genome Biol. 2014;15:422. https://doi.org/10.1186/s13059-014-0422-2.

23. Lois LM. Diversity of the SUMOylation machinery in plants. Biochem Soc Trans. 2010;38:60-4. https://doi.org/10.1042/BST0380060.

24. Bernier-Villamor V, Sampson DA, Matunis MJ, Lima CD. Structural basis for E2-mediated SUMO conjugation revealed by a complex between ubiquitinconjugating enzyme Ubc9 and RanGAP1. Cell. 2002;108:345-56. https://doi. org/10.1016/S0092-8674(02)00630-X.

25. Song J, Durrin LK, Wilkinson TA, Krontiris TG, Chen Y. Identification of a SUMO-binding motif that recognizes SUMO-modified proteins. Proc Natl Acad Sci. 2004;101:14373-8. https://doi.org/10.1073/pnas.0403498101.

26. Beauclair G, Bridier-Nahmias A, Zagury J-F, Saïb A, Zamborlini A. JASSA: a comprehensive tool for prediction of SUMOylation sites and SIMs. Bioinformatics. 2015;31:3483-91. https://doi.org/10.1093/bioinformatics/btv403.

27. Kerscher O. SUMO junction-what's your function? EMBO Rep. 2007;8:5505. https://doi.org/10.1038/sj.embor.7400980.

28. Parker JL, Bucceri A, Davies AA, Heidrich K, Windecker H, Ulrich HD. SUMO modification of PCNA is controlled by DNA. EMBO J. 2008;27:2422-31. https://doi.org/10.1038/emboj.2008.162.

29. Lee J, Nam J, Park HC, Na G, Miura K, Jin JB, et al. Salicylic acid-mediated innate immunity in Arabidopsis is regulated by SIZ1 SUMO E3 ligase. Plant J. 2006;49:79-90. https://doi.org/10.1111/j.1365-313X.2006.02947.x.

30. Cheng $X$, Xiong R, Li Y, Li F, Zhou X, Wang A. Sumoylation of turnip mosaic virus RNA polymerase promotes viral infection by counteracting the host NPR1-mediated immune response. Plant Cell. 2017;29:508-25. https://doi. org/10.1105/tpc.16.00774.

31. Cui X, Li G, Wang D, Hu D, Zhou X. A Begomovirus DNA-encoded protein binds DNA, functions as a suppressor of RNA silencing, and targets the cell nucleus. J Virol. 2005;79:10764-75. https://doi.org/10.1128/JVI.79.16.1076410775.2005

32. Covey SN, Al-Kaff NS, Lángara A, Turner DS. Plants combat infection by gene silencing. Nature. 1997;385:781-2. https://doi.org/10.1038/385781a0.

33. Das S, Hegde A, Shivaprasad PV. Molecular characterization of a new begomovirus infecting Synedrella nodiflora in South India. Arch Virol. 2018; $163: 2551-4$

34. Zhao Q, Xie Y, Zheng Y, Jiang S, Liu W, Mu W, et al. GPS-SUMO: a tool for the prediction of sumoylation sites and SUMO-interaction motifs. Nucleic Acids Res. 2014;42:W325-30. https://doi.org/10.1093/nar/gku383.

35. Matic I, Schimmel J, Hendriks IA, van Santen MA, van de Rijke F, van Dam H, et al. Site-specific identification of SUMO-2 targets in cells reveals an inverted SUMOylation motif and a hydrophobic cluster SUMOylation motif. Mol Cell. 2010;39:641-52. https://doi.org/10.1016/j.molcel.2010.07.026.

36. Amin I, Patil BL, Briddon RW, Mansoor S, Fauquet CM. Comparison of phenotypes produced in response to transient expression of genes encoded by four distinct begomoviruses in Nicotiana benthamiana and their correlation with the levels of developmental miRNAs. Virol J. 2011;8: 238. https://doi.org/10.1186/1743-422X-8-238.

37. Bazzini AA, Hopp HE, Beachy RN, Asurmendi S. Infection and coaccumulation of tobacco mosaic virus proteins alter microRNA levels, correlating with symptom and plant development. Proc Natl Acad Sci. 2007; 104:12157-62. https://doi.org/10.1073/pnas.0705114104.

38. Colby T, Matthäi A, Boeckelmann A, Stuible H-P. SUMO-conjugating and SUMO-deconjugating enzymes from Arabidopsis. Plant Physiol. 2006;142: 318-32. https://doi.org/10.1104/pp.106.085415.

39. Kurepa J, Walker JM, Smalle J, Gosink MM, Davis SJ, Durham TL, et al. The small ubiquitin-like modifier (SUMO) protein modification system in Arabidopsis. J Biol Chem. 2003;278:6862-72. https://doi.org/10.1074/jbc. M209694200.

40. Brückner A, Polge C, Lentze N, Auerbach D, Schlattner U. Yeast two-hybrid, a powerful tool for systems biology. Int J Mol Sci. 2009;10:2763-88. https:// doi.org/10.3390/ijms10062763.
41. Dahlmann B, Kuehn L, Rutschmann M, Reinauer H. Purification and characterization of a multicatalytic high-molecular-mass proteinase from rat skeletal muscle. Biochem J. 1985;228:161-70. https://doi.org/10.1042/ bj2280161.

42. Lange OF, Rossi P, Sgourakis NG, Song Y, Lee H-W, Aramini JM, et al. Determination of solution structures of proteins up to $40 \mathrm{kDa}$ using CSRosetta with sparse NMR data from deuterated samples. Proc Natl Acad Sci. 2012;109:10873-8. https://doi.org/10.1073/pnas.1203013109.

43. Tripathi V, Chatterjee KS, Das R. Casein kinase-2-mediated phosphorylation increases the SUMO-dependent activity of the cytomegalovirus transactivator IE2. J Biol Chem. 2019;294:14546-61. https://doi.org/10.1074/ jbc.RA119.009601.

44. Eini O, Dogra S, Selth LA, Dry IB, Randles JW, Rezaian MA. Interaction with a host ubiquitin-conjugating enzyme is required for the pathogenicity of a geminiviral DNA $\beta$ satellite. Mol Plant-Microbe Interact. 2009;22:737-46.

45. Bhattacharyya D, Gnanasekaran P, Kumar RK, Kushwaha NK, Sharma VK, Yusuf MA, et al. A geminivirus betasatellite damages the structural and functional integrity of chloroplasts leading to symptom formation and inhibition of photosynthesis. J Exp Bot. 2015;66:5881-95.

46. Shen W, Dallas MB, Goshe MB, Hanley-Bowdoin L. SnRK1 phosphorylation of AL2 delays cabbage leaf curl virus infection in Arabidopsis. J Virol. 2014;88: 10598-612.

47. Li H, Zeng R, Chen Z, Liu X, Cao Z, Xie Q, et al. S-acylation of a geminivirus C4 protein is essential for regulating the CLAVATA pathway in symptom determination. J Exp Bot. 2018;69:4459-68. https://doi.org/10.1093/jxb/ery228.

48. Kushwaha NK, Bhardwaj M, Chakraborty S. The replication initiator protein of a geminivirus interacts with host monoubiquitination machinery and stimulates transcription of the viral genome. PLoS Pathog. 2017;13: e1006587. https://doi.org/10.1371/journal.ppat.1006587.

49. Chowda-Reddy RV, Achenjang F, Felton C, Etarock MT, Anangfac M-T, Nugent $P$, et al. Role of a geminivirus AV2 protein putative protein kinase $C$ motif on subcellular localization and pathogenicity. Virus Res. 2008;135:11524. https://doi.org/10.1016/j.virusres.2008.02.014.

50. Tsutakawa SE, Yan C, Xu X, Weinacht CP, Freudenthal BD, Yang K, et al. Structurally distinct ubiquitin- and Sumo-modified PCNA: implications for their distinct roles in the DNA damage response. Structure. 2015;23:724-33. https://doi.org/10.1016/j.str.2015.02.008.

51. Sianipar IR, Matsui C, Minami N, Gan X, Deng L, Hotta H, et al. Physical and functional interaction between hepatitis C virus NS5A protein and ovarian tumor protein deubiquitinase 7B. Microbiol Immunol. 2015;59:466-76. https://doi.org/10.1111/1348-0421.12278.

52. Domingues $\mathrm{P}$, Golebiowski F, Tatham MH, Lopes AM, Taggart A, Hay RT et al. Global reprogramming of host SUMOylation during influenza virus infection. Cell Rep. 2015;13:1467-80. https://doi.org/10.1016/j.celrep.2015. 10.001 .

53. Boggio R, Colombo R, Hay RT, Draetta GF, Chiocca S. A mechanism for inhibiting the SUMO pathway. Mol Cell. 2004;16:549-61. https://doi.org/10. 1016/j.molcel.2004.11.007.

54. De La Cruz-Herrera CF, Shire K, Siddiqi UZ, Frappier L. A genome-wide screen of Epstein-Barr virus proteins that modulate host SUMOylation identifies a SUMO E3 ligase conserved in herpesviruses. PLoS Pathog. 2018; 14:e1007176. https://doi.org/10.1371/journal.ppat.1007176.

55. Sanchez-Duran MA, Dallas MB, Ascencio-lbanez JT, Reyes MI, Arroyo-Mateos $M$, Ruiz-Albert J, et al. Interaction between geminivirus replication protein and the SUMO-conjugating enzyme is required for viral infection. J Virol. 2011;85:9789-800

56. Rytz TC, Miller MJ, McLoughlin F, Augustine RC, Marshall RS, Juan Y, et al. SUMOylome Profiling Reveals a Diverse Array of Nuclear TargetsModified by the SUMO Ligase SIZ1 during Heat Stress. Plant Cell. 2018;30:1077-99. https://doi.org/10.1105/tpc.17.00993.

57. Murtas G, Reeves PH, Fu YF, Bancroft I, Dean C, Coupland G. A Nuclear Protease Required for Flowering-Time Regulation in Arabidopsis Reduces the Abundance of SMALL UBIQUITIN-RELATED MODIFIER Conjugates. Plant Cell. 2003;15:2308-19.

58. Gutierrez C. Geminivirus DNA replication. Cell Mol Life Sci. 1999;56:313-29. https://doi.org/10.1007/s000180050433.

59. Zhao J, Liu Q, Zhang H, Jia Q, Hong Y, Liu Y. The Rubisco small subunit is involved in Tobamovirus movement and Tm-2 2-mediated extreme resistance. Plant Physiol. 2013;161:374-83. https://doi.org/10.1104/pp.112.209213.

60. Chang C-C, Naik MT, Huang Y-S, Jeng J-C, Liao P-H, Kuo H-Y, et al. Structural and functional roles of Daxx SIM phosphorylation in SUMO Paralog-selective 
binding and apoptosis modulation. Mol Cell. 2011;42:62-74. https://doi.org/ 10.1016/j.molcel.2011.02.022.

61. Chatterjee KS, Tripathi V, Das R. A conserved and buried edge-to-face aromatic interaction in small ubiquitin-like modifier (SUMO) has a role in SUMO stability and function. J Biol Chem. 2019;294:6772-84. https://doi.org/ 10.1074/jbc.RA118.006642.

62. Sunilkumar G, Vijayachandra K, Veluthambi K. Preincubation of cut tobacco leaf explants promotes Agrobacterium-mediated transformation by increasing vir gene induction. Plant Sci. 1999;141:51-8. https://doi.org/10. 1016/S0168-9452(98)00228-3.

63. Stachel SE, Zambryski PC. Agrobacterium tumefaciens and the susceptible plant cell: a novel adaptation of extracellular recognition and DNA conjugation. Cell. 1986;47:155-7. https://doi.org/10.1016/00928674(86)90437-X.

64. Yanofsky MF, Porter SG, Young C, Albright LM, Gordon MP, Nester EW. The virD operon of Agrobacterium tumefaciens encodes a site-specific endonuclease. Cell. 1986:47:471-7. https://doi.org/10.1016/00928674(86)90604-5.

65. Wang W, Vignani R, Scali M, Cresti M. A universal and rapid protocol for protein extraction from recalcitrant plant tissues for proteomic analysis. Electrophoresis. 2006;27:2782-6. https://doi.org/10.1002/elps.200500722.

66. Tirumalai V, Swetha C, Nair A, Pandit A, Shivaprasad PV. miR828 and miR858 regulate VVMYB114 to promote anthocyanin and flavonol accumulation in grapes. J Exp Bot. 2019;70:4775-92. https://doi.org/10.1093/jxb/erz264.

67. Shivaprasad PV, Rajeswaran R, Blevins T, Schoelz J, Meins F, Hohn T, et al. The CaMV transactivator/viroplasmin interferes with RDR6-dependent transacting and secondary siRNA pathways in Arabidopsis. Nucleic Acids Res. 2008:36:5896-909. https://doi.org/10.1093/nar/gkn590.

68. Shivaprasad PV, Thomas M, Balamani V, Biswas D, Vanitharani R, Karthikeyan AS, et al. Factors contributing to deletion within Mungbean yellow mosaic virus partial dimers in binary vectors used for agroinoculation. J Virol Methods. 2006;137:72-81. https://doi.org/10.1016/j.jviromet.2006.06.002.

69. Rogers SO, Bendich AJ. Extraction of total cellular DNA from plants, algae and fungi. In: Plant molecular biology manual. Dordrecht: Springer Netherlands; 1994. p. 183-90. https://doi.org/10.1007/978-94-011-0511-8_12.

70. Shivaprasad PV, Thillaichidambaram P, Balaji V, Veluthambi K. Expression of full-length and truncated Rep genes from Mungbean yellow mosaic virusVigna inhibits viral replication in transgenic tobacco. Virus Genes. 2006;33: 365-74.

71. Gietz RD, Woods RA. Transformation of yeast by lithium acetate/singlestranded carrier DNA/polyethylene glycol method. Methods Enzymol. 2002; 350:87-96

72. Delaglio F, Grzesiek S, Vuister G, Zhu G, Pfeifer J, Bax A. NMRPipe: a multidimensional spectral processing system based on UNIX pipes. J Biomol NMR. 1995;6. https://doi.org/10.1007/BF00197809.

73. Lee W, Tonelli M, Markley JL. NMRFAM-SPARKY: enhanced software for biomolecular NMR spectroscopy. Bioinformatics. 2015;31:1325-7. https://doi. org/10.1093/bioinformatics/btu830

74. Bahrami A, Assadi AH, Markley JL, Eghbalnia HR. Probabilistic interaction network of evidence algorithm and its application to complete labeling of peak lists from protein NMR spectroscopy. PLoS Comput Biol. 2009;5: e1000307.

75. Ulrich EL, Akutsu H, Doreleijers JF, Harano Y, loannidis YE, Lin J, et al. BioMagResBank. Nucleic Acids Res. 2007;36Database:D402-8.https://doi.org/ 10.1093/nar/gkm957.

\section{Publisher's Note}

Springer Nature remains neutral with regard to jurisdictional claims in published maps and institutional affiliations.

Ready to submit your research? Choose BMC and benefit from:

- fast, convenient online submission

- thorough peer review by experienced researchers in your field

- rapid publication on acceptance

- support for research data, including large and complex data types

- gold Open Access which fosters wider collaboration and increased citations

- maximum visibility for your research: over $100 \mathrm{M}$ website views per year

At $\mathrm{BMC}$, research is always in progress.

Learn more biomedcentral.com/submissions 